\title{
Stability and Instability Matrices for Linear Evolution Variational Inequalities
}

\author{
Daniel Goeleven and Bernard Brogliato
}

\begin{abstract}
This paper deals with the characterization of the stability and instability matrices for a class of unilaterally constrained dynamical systems, represented as linear evolution variational inequalities (LEVI). Such systems can also be seen as a sort of differential inclusion, or (in special cases) as linear complementarity systems, which in turn are a class of hybrid dynamical systems. Examples show that the stability of the unconstrained system and that of the constrained system, may drastically differ. Various criteria are proposed to characterize the stability or the instability of LEVI.
\end{abstract}

Index Terms-Convex analysis, copositive matrices, hybrid dynamics, instability matrices, Lyapunov stability, stability matrices, unilateral constraints, variational inequalities.

\section{INTRODUCTION}

$\mathbf{T}$ HE stability of stationary solutions of dynamic systems constitutes a very important topic in applied mathematics and engineering. It is well-known that in the case of a large class of nonlinear differential equations the spectrum of "linearized" operators determines the Lyapunov stability of an equilibrium. This is known as the Lyapunov's linearization method [31, Sec. 5.5]. However, many important problems in engineering (see [16], [22]-[24], [28]) involve inequalities in their mathematical formulation and consequently possess intrinsic nonsmoothness. This is for instance the case of complementarity dynamical systems [16]-[18], whose study still presents many open challenging problems [5]. For these last cases the question of stability is much more complicated to be investigated, as it is the case in general for hybrid dynamical systems, see e.g., [8], [9], [34]-[36]. An interesting class of unilaterally constrained dynamical systems can be represented under the formalism of evolution variational inequalities; see, e.g., [20]. Roughly speaking (a rigorous definition will be given next), evolution variational inequalities are a special type of dynamical systems whose state is forced to remain in a set $K \subseteq \mathbb{R}^{n}$ (i.e., the ambient state space is $\mathbb{R}^{n}$ ). When the state attains the boundary of $K$, then the vector field is suitably modified. Such dynamical systems are therefore nonsmooth and nonlinear (the only case in which the dynamics may be linear is the degenerate case $K=\mathbb{R}^{n}$, which is of no interest here). Evolution variational inequalities are widely used in applied mathematics and various fields of science with applica-

Manuscript received December 4, 2002; revised September 10, 2003. Recommended by Associate Editor R. Freeman. This work was supported in part by the European project SICONOS IST 2001-37172.

D. Goeleven is with the IREMIA, University of La Réunion, Saint-Denis 97400, France (e-mail: Daniel.Goeleven@univ-reunion.fr).

B. Brogliato is with the INRIA Rhônes-Alpes, Saint-Ismier 38334, France (e-mail: Bernard.Brogliato@inrialpes.fr)

Digital Object Identifier 10.1109/TAC.2004.825654 tions to behavior of oligopolistic markets, urban transportation networks, traffic networks, international trade, agricultural and energy markets (spatial price equilibria) [37]-[39], [41], [42], [44]. In fact, the research efforts made to model the cited physical systems are relatively new, and are meant to extend the static variational inequalities [43] in order to incorporate dynamical effects. This is often called disequilibrium models in the related literature [41], whose trajectories should converge to the equilibrium that is a solution of a static variational inequality. Though such models often are of the infinite-dimensional type, suitable discretization may recast them into finite-dimensional evolution variational inequalities [42]. Let us briefly present the dynamical systems we will deal with and the relationships with complementarity systems and differential inclusions.

The Class of Dynamical Systems: Let $K \subset \mathbb{R}^{n}$ be a nonempty closed convex set. Let $A \in \mathbb{R}^{n \times n}$ be a given matrix and $F: \mathbb{R}^{n} \rightarrow \mathbb{R}^{n}$ a nonlinear operator. For $\left(t_{0}, x_{0}\right) \in \mathbb{R} \times K$, we consider the problem $P\left(t_{0}, x_{0}\right)$ : Find a function $t \rightarrow x(t)\left(t \geq t_{0}\right)$ with $x \in C^{0}\left(\left[t_{0},+\infty\right) ; \mathbb{R}^{n}\right)$, $(d x / d t) \in L_{\mathrm{loc}}^{\infty}\left(t_{0},+\infty ; \mathbb{R}^{n}\right)$ and such that (1), as shown at the bottom of the next page, holds.

Here $\langle.,$.$\rangle denotes the euclidean scalar product in \mathbb{R}^{n}$. The corresponding norm is denoted by $\|\cdot\|$. The system in (1) is an evolution variational inequality which we denote as $\operatorname{LEVI}(A, K)$ when $F \equiv 0$. It follows from standard convex analysis that (1) can be rewritten equivalently as the differential inclusion

$$
\begin{cases}\frac{d x}{d t}(t)+A x(t)+F(x(t)) \in-N_{K}(x(t)) & \\ x(t) \in K, & t \geq t_{0}\end{cases}
$$

where $N_{K}(x(t))=\left\{s \in \mathbb{R}^{n}:\langle s, v-x(t)\rangle \leq 0, \forall v \in K\right\}$ is the normal cone to $K$ at $x(t)$ [19]. In case $K=\left\{x \in \mathbb{R}^{n}\right.$ : $C x+d \geq 0\}$ for some matrix $C \in \mathbb{R}^{m \times n}$ and vector $d \in \mathbb{R}^{m}$, we can rewrite (1) as

$$
\left\{\begin{array}{l}
\frac{d x}{d t}(t)+A x(t)+F(x(t))=C^{T} \lambda \\
0 \leq y=C x(t)+d \perp \lambda \geq 0
\end{array}\right.
$$

where $\lambda \in \mathbb{R}^{m}$ is a Lagrange multiplier, and the second line of (3) means that both $y$ and $\lambda$ have to be nonnegative, and orthogonal. System (3) belongs to the class of linear complementarity systems (LCSs) with a relative degree $r_{y \lambda} \geq 1$ between $y$ and $\lambda[16]-[18]$. Variational inequalities and complementarity are known to be closely related [32], [33], [37].

Remark 1:

- Mechanical systems with unilateral constraints do not belong to the class of systems studied in this paper. Indeed in (3) one has $y=D x+d$ with $D \neq C$ for mechanical systems. 
- The problems related to positively invariant sets and control holdability of sets (see, e.g., [1], [2], and [29]) are essentially different from what is studied in this paper. Indeed (see theorem 1 below) trajectories of (1) remain in $K$ for all $A$ and all $t \geq t_{0}$ because the dynamics is modified on the boundary of $K$.

- Assume that $K=\left\{x \in \mathbb{R}^{n}: g(x) \geq 0\right\}$ for some convex function $g(\cdot)$. Then $\partial K$ may possess an infinity of corners. The framework in (1) encompasses such cases.

The stability of evolution variational inequalities has been investigated by various authors; see, e.g., [6], [10]-[12], [40], and [45]. It is well-known that an EVI as in (1) can be stable when it is not constrained $\left(K=\mathbb{R}^{n}\right)$, whereas it becomes unstable for some $K \subset \mathbb{R}^{n}$, and vice-versa [40, Ex. 2.1 and 2.2]. In particular this means that studying the stability of an EVI from the spectrum of the Jacobian of its unconstrained dynamics (in (1) this is $\left.(\partial(A x+F(x)) /(\partial x)) \in \mathbb{R}^{n \times n}\right)$, is generally hopeless: the tangent linearization of an unconstrained system may be exponentially stable, while the constrained system (i.e., the evolution variational inequality) is unstable. On the contrary, the tangent linearization of the unconstrained dynamics may be exponentially unstable while the constrained system is asymptotically stable. It is consequently of great interest to study the stability of such unilaterally constrained dynamical systems. Perhaps the first result on stability of EVI in the framework of transportation sciences is in [44], who investigated the stability of Wardropean equilibrium under a dynamical system constructed to describe the drivers' behavior in changing their route choice. The results in [40] and [45] concern projected dynamical systems on polyhedra (which can be written as complementarity systems in (3) [37]). They characterize the attractivity and stability of stationary solutions under monotonicity of the vector field [corresponding to $A x+F(x)$ in (1)]. The results in [6] generalize those in [10]-[12]. They provide general conditions under which the second method of Lyapunov extends to EVIs.

The main achievements of this paper are the following. Starting from the general results on the Lyapunov stability of evolution variational inequalities in [6], 1) we show how copositive matrices on $K$ can be used to construct Lyapunov functions for linear evolution variational inequalities; 2) we propose several practical criteria to test the stability; 3) we present examples (the absolute stability problem, electrical circuits) which were not previously studied in this framework and, thus, bring original applications to the field. Compared to the cited works on the stability of variational inequalities, our work focuses on $\operatorname{LEVI}(A, K)$ (which does not at all mean that the systems we deal with are linear), and we provide a thorough analysis of this particular case.

Let us note that in parallel to the above-mentioned studies on stability of various classes of hybrid dynamical systems, the stability properties of special classes of matrices (like so-called
P-matrices or M-matrices [21]) is still an active research area [7]. Though the objective of these studies differ from ours (especially, the systems studied in this paper are not only nonsmooth, but nonlinear), it is interesting to notice that the analytical tools they use have strong similarities with some of the tools used in this paper. Therefore this work can also be seen as a new branch in the field of matrix stability analysis.

In Section II, some theoretical results concerning the wellposedness, stability definitions and an extension of the second method of Lyapunov are presented. In Section III matrices $A$ in (1) that yield stable systems are studied, as well as criteria to characterize unstable matrices $A$. Section IV concerns stable and unstable $\operatorname{LEVI}(A, K)$. Section $\mathrm{V}$ shows that stability is preserved under small nonlinear disturbances. Section VI is devoted to present various criteria which allow to test the stability or the instability of matrices $A$; many examples illustrate the developments. Section VII applies the results to some concrete problems: an extension of the absolute stability problem, and a class of dissipative linear complementarity problems (which models some electrical circuits with ideal diodes). Conclusions end the paper.

\section{AbStRact Results}

Let us first specify some conditions ensuring the existence and uniqueness of the initial value problem $P\left(t_{0}, x_{0}\right)$. The following existence and uniqueness result is a direct consequence of [6, Cor. 2.2].

Theorem 1: Let $K$ be a nonempty closed convex subset of $\mathbb{R}^{n}$ and let $A \in \mathbb{R}^{n \times n}$ be a real matrix of order $n$. Suppose that $F: \mathbb{R}^{n} \rightarrow \mathbb{R}^{n}$ can be written as

$$
F=F_{1}+\Phi^{\prime}
$$

where $F_{1}$ is Lipschitz continuous and $\Phi \in C^{1}\left(\mathbb{R}^{n} ; \mathbb{R}\right)$ is convex. Let $t_{0} \in \mathbb{R}$ and $x_{0} \in K$ be given. Then there exists a unique $x \in C^{0}\left(\left[t_{0},+\infty\right) ; \mathbb{R}^{n}\right)$ such that

$$
\begin{aligned}
& \frac{d x}{d t} \in L_{\mathrm{loc}}^{\infty}\left(\left[t_{0},+\infty\right) ; \mathbb{R}^{n}\right) \\
& x \text { is right-differentiable on }\left[t_{0},+\infty\right) \\
& x\left(t_{0}\right)=x_{0} \\
& x(t) \in K, \quad t \geq t_{0} \\
& \left\langle\frac{d x}{d t}(t)+A x(t)+F(x(t)), v-x(t)\right\rangle \geq 0 \\
& \forall v \in K, \text { a.e. } t \geq t_{0} .
\end{aligned}
$$

Suppose that the assumptions of Theorem 1 are satisfied and denote by $x\left(\cdot ; t_{0}, x_{0}\right)$ the unique solution of problem $P\left(t_{0}, x_{0}\right)$. Suppose now in addition that

$$
0 \in K
$$

$$
\begin{cases}x(t) \in K, & t \geq t_{0} \\ \left\langle\frac{d x}{d t}(t)+A x(t)+F(x(t)), v-x(t)\right\rangle \geq 0, & \forall v \in K, \text { a.e. } t \geq t_{0} \\ x\left(t_{0}\right)=x_{0} & \end{cases}
$$


and

$$
-F(0) \in N_{K}(0)
$$

that is

$$
\langle F(0), h\rangle \geq 0 \quad \forall h \in K \text {. }
$$

Then

$$
x\left(t ; t_{0}, 0\right)=0, \quad t \geq t_{0}
$$

i.e., the trivial solution 0 is the unique solution of problem $P\left(t_{0}, 0\right)$.

Remark 2: The wellposedness results of Theorem 1 continue to hold for a controlled $\operatorname{LEVI}(A, B, K)$ defined as $\langle(d x / d t)(t)+A x(t)+B u(t)+F(x(t)), v-x(t)\rangle \geq 0$, $\forall v \in K$, with $B \in \mathbb{R}^{m \times n}, u \in C^{0}\left(\left[t_{0},+\infty\right) ; \mathbb{R}^{m}\right)$ and $(d u / d t) \in L_{\text {loc }}^{1}\left(\left[t_{0},+\infty\right) ; \mathbb{R}^{m}\right)$; see [6]. This is important in view of controllability issues. In relationship with (2) and (3), let us notice that if $y=C x+d+B u(t)$ in (3), then the right-hand side of (2) becomes equal to $N_{K(u(t))}(x(t))$, i.e., the convex set $K(u(t))=\left\{x \in \mathbb{R}^{n}: C x+d+B u(t) \geq 0\right\}$ is time varying. If $A=0$ and $F(\cdot)=0$, then the obtained system fits within Moreau's sweeping process [27]. This case is not studied in this paper. However, some electrical circuits possess a relative degree 0 between $y$ and $u$ [17], so the extension of the presented results toward convex sets $K(t)$ is valuable.

We may now define as in [6] the stability of the trivial solution. The stationary solution 0 is called stable if small perturbations of the initial condition $x\left(t_{0}\right)=0$ lead to solutions which remain in the neighborhood of 0 for all $t \geq t_{0}$, precisely, the following.

Definition 1: The equilibrium point $x=0$ is said to be stable in the sense of Lyapunov if for every $\varepsilon>0$ there exists $\eta=$ $\eta(\varepsilon)>0$ such that for any $x_{0} \in K$ with $\left\|x_{0}\right\| \leq \eta$ the solution $x\left(\cdot ; t_{0}, x_{0}\right)$ of problem $P\left(t_{0}, x_{0}\right)$ satisfies $\left\|x\left(t ; t_{0}, x_{0}\right)\right\| \leq \varepsilon$, $\forall t \geq t_{0}$.

If in addition the trajectories of the perturbed solutions are attracted by 0 then we say that the stationary solution is asymptotically stable, precisely, the following..

Definition 2: The equilibrium point $x=0$ is asymptotically stable if it is stable and there exists $\delta>0$ such that for any $x_{0} \in K$ with $\left\|x_{0}\right\| \leq \delta$ the solution $x\left(\cdot ; t_{0}, x_{0}\right)$ of problem $P\left(t_{0}, x_{0}\right)$ fulfills

$$
\lim _{t \rightarrow+\infty}\left\|x\left(t ; t_{0}, x_{0}\right)\right\|=0 .
$$

The notion of instability is now given as the negation of Definition 1 .

Definition 3: The equilibrium point $x=0$ is unstable if it is not stable, i.e., there exists $\varepsilon>0$ such that for any $\eta>0$, one may find $x_{0} \in K$ with $\left\|x_{0}\right\| \leq \eta$ and $\bar{t} \geq t_{0}$ such that the solution $x\left(\cdot ; t_{0}, x_{0}\right)$ of problem $P\left(t_{0}, x_{0}\right)$ verifies

$$
\left\|x\left(\bar{t} ; t_{0}, x_{0}\right)\right\|>\varepsilon \text {. }
$$

Let us now give general abstract theorems of stability, asymptotic stability and instability in terms of generalized Lyapunov functions $V \in C^{1}\left(\mathbb{R}^{n} ; \mathbb{R}\right)$. The following results are particular cases of the ones proved in [6].
Theorem 2: Suppose that the assumptions of Theorem 1 together with condition (10) hold. Suppose that there exist $\sigma>0$ and $V \in C^{1}\left(\mathbb{R}^{n} ; \mathbb{R}\right)$ such that

1)

$$
V(x) \geq a(\|x\|), \quad x \in K, \quad\|x\| \leq \sigma
$$

with $a:[0, \sigma] \rightarrow \mathbb{R}$ satisfying $a(t)>0, \forall t \in(0, \sigma)$;

2) $V(0)=0$;

3) $x-V^{\prime}(x) \in K, x \in \partial K,\|x\| \leq \sigma$

4) $\left\langle A x+F(x), V^{\prime}(x)\right\rangle \geq 0, x \in \bar{K},\|x\| \leq \sigma$.

Then, the trivial solution of (7) and (8) is stable.

Theorem 3: Suppose that the assumptions of Theorem 1, together with (10), hold. Suppose that there exist $\lambda>0, \sigma>0$ and $V \in C^{1}\left(\mathbb{R}^{n} ; \mathbb{R}\right)$ such that

1)

$$
V(x) \geq a(\|x\|), \text { for all } x \in K,\|x\| \leq \sigma
$$

with $a:[0, \sigma] \rightarrow \mathbb{R}$ satisfying $a(t) \geq c t^{\tau}, \forall t \in[0, \sigma]$, for some constants $c>0, \tau>0$;

2) $V(0)=0$;

3) $x-V^{\prime}(x) \in K$, for all $x \in \partial K,\|x\| \leq \sigma$;

4) $\left\langle A x+F(x), V^{\prime}(x)\right\rangle \geq \lambda V(x)$, for all $x \in K,\|x\| \leq \sigma$. Then, the trivial solution of (7) and (8) is asymptotically stable.

Let us note that (3) is a sufficient condition which implies $-V^{\prime}(x) \in T_{K}(x)$ for all $x \in \partial K,\|x\| \leq \sigma$, where $T_{K}(x)$ is the tangent cone to $K$ at $x$, and $V^{\prime}(x)$ denotes the gradient of $V(\cdot)$ at $x$ [19, Prop. 5.2.1]. Therefore, it characterizes the orientation of the level sets of $V(\cdot)$ with respect to the boundary of $K$. If the conditions of Theorem 3 hold on $\mathbb{R}^{n}$ [i.e., $\sigma=+\infty$ in (1), (3), and (4)], the trivial solution of (7) and (8) is globally attractive in $K$. We formulate now an instability result.

Theorem 4: Suppose that the assumptions of Theorem 1 together with condition (10) hold. Suppose also that $K \backslash\{0\} \neq \emptyset$. If there exist $V \in C^{1}\left(\mathbb{R}^{n} ; \mathbb{R}\right)$ and $\alpha>0$ such that

1)

$$
V(x) \leq b(\|x\|), \quad x \in K
$$

with $b:[0,+\infty) \rightarrow \mathbb{R}$ satisfying $b(t) \leq k t^{s}, \forall t \geq 0$, for some constants $k>0, s>0$;

2) $V(x)>0, x \in K, x \neq 0$, near 0 ;

3) $V^{\prime}(x) \in K_{\infty}, x \in \partial K$;

4) $\left\langle A x+F(x), V^{\prime}(x)\right\rangle \leq-\alpha V(x), x \in K$;

then the trivial solution (7) and (8) is unstable.

The recession cone $K_{\infty}$ will be defined in Section III. We note that Theorems 2-4 provide sufficient conditions for stability and instability. The same will apply for Definitions 4-6 in Section III.

\section{Stability And Instability Matrices on A Closed CONVEX SET}

The aim of this section is to introduce some classes of stability and instability matrices. The results obtained here will be used later in this paper to construct generalized Lyapunov functions needed to apply the abstract stability results given in Section II. Let us first recall some basic tools from convex analysis [19]. 
Let $C$ be a nonempty subset of $\mathbb{R}^{n}$. We say that $C$ is a cone if $\lambda C \subset C, \forall \lambda>0$. A nonempty closed convex cone is characterized by the relations

$$
\lambda C \subset C \quad \forall \lambda>0 \quad C+C \subset C, \quad 0 \in C .
$$

Let $D \subset \mathbb{R}^{n}$ be a nonempty closed convex set. The recession (or asymptotic) cone $D_{\infty}$ of $D$ is defined by

$$
D_{\infty}=\cap_{\lambda>0} \lambda\left(D-x_{0}\right)
$$

where $x_{0}$ is an arbitrary fixed element of $D$. In other words the recession cone of $D$ is the set of directions from which one can go straight from any point $x_{0} \in D$ to infinity, while staying in $D$ [19, Sec. A 2.2]. The set $D_{\infty}$ is a closed convex cone. One has

$$
D+D_{\infty} \subset D
$$

and thus, if $0 \in D$ then $D_{\infty} \subset D$. If $D$ is symmetric, i.e., $x \in D \Rightarrow-x \in D$ then clearly $D_{\infty}$ is a subspace of $\mathbb{R}^{n}$. If $D$ is bounded then $D_{\infty}=\{0\}$. For $x \in \mathbb{R}^{n}$ we denote by $P_{D}(x)$ the unique solution of the variational inequality: Find $z \in D$, such that

$$
\langle z-x, y-z\rangle \geq 0 \quad \forall y \in D \text {. }
$$

The mapping $P_{D}: \mathbb{R}^{n} \rightarrow D ; x \mapsto P_{D}(x)$ is called the projection on $D$. If $D$ is a subspace of $\mathbb{R}^{n}$ then $P_{D}$ is linear and symmetric. In this case, we write $P_{D}(x)=P_{D} x$ where $P_{D} \in \mathbb{R}^{n \times n}$ denotes the symmetric matrix of orthogonal projection on $D$.

In the sequel, $A \in \mathbb{R}^{n \times n}$ denotes a real matrix of order $n$ and $K \subset \mathbb{R}^{n \times n}$ is a subset of $\mathbb{R}^{n}$. Let us also point out the following notations: $\sigma(A)$ denotes the spectrum of $A, \rho(A)$ is the spectral radius of $A$ and for $\lambda \in \sigma(A), E_{A}(\lambda)$ denotes the corresponding eigenspace. The transpose of $A$ is denoted by $A^{T}, \operatorname{ker}\{A\}$ is the null space of $A$ and $\operatorname{tr}\{A\}$ is the trace of $A$. The Kronecker product of two matrices $A \in \mathbb{R}^{n \times l}$ and $B \in$ $\mathbb{R}^{m \times k}$ is denoted as $\otimes$ and $A \otimes B \in \mathbb{R}^{m n \times l k}$ [26]. The interior and boundary of $K$ are respectively denoted by $\stackrel{\circ}{K}$ and $\partial K$. We assume that

$\left(H_{1}\right) K$ is closed;

$\left(H_{2}\right) K$ is convex;

$\left(H_{3}\right) \quad 0 \in K$.

Only the possible additional conditions needed on $K$ will be specified in this paper.

Definition 4: The matrix $A \in \mathbb{R}^{n \times n}$ is Lyapunov positive stable on $K$ if there exists a matrix $G \in \mathbb{R}^{n \times n}$ such that

1) $\inf _{x \in K \backslash\{0\}}\left(\langle G x, x\rangle /\|x\|^{2}\right)>0$;

2) $\left\langle A x,\left[G+G^{T}\right] x\right\rangle \geq 0, \forall x \in K$;

3) $x \in \partial K \Rightarrow\left[I-\left[G+G^{T}\right]\right] x \in K$

Definition 5: The matrix $A \in \mathbb{R}^{n \times n}$ is Lyapunov positive strictly-stable on $K$ if there exists a matrix $G \in \mathbb{R}^{n \times n}$ such that

1) $\inf _{x \in K \backslash\{0\}}\left(\langle G x, x\rangle /\|x\|^{2}\right)>0$;

2) $\inf _{x \in K \backslash\{0\}}\left(\left\langle A x,\left[G+G^{T}\right] x\right\rangle /\|x\|^{2}\right)>0$;

3) $x \in \partial K \Rightarrow\left[I-\left[G+G^{T}\right]\right] x \in K$.
Stable matrices are sometimes called semistable, whereas strictly-stable matrices are sometimes called stable [7].

Remark 3: Condition (1) of Definition 4 (and 5) is equivalent to the existence of a constant $c>0$ such that

$$
\langle G x, x\rangle \geq c\|x\|^{2} \quad \forall x \in K .
$$

Indeed, set

$$
C:=\inf _{x \in K \backslash\{0\}} \frac{\langle G x, x\rangle}{\|x\|^{2}} .
$$

If $+\infty>C>0$ then it is clear that (11) holds with $c=C$. If $C=+\infty$, then necessarily $K=\{0\}$ and the relation in (11) is trivial. On the other hand, it is clear that if (11) holds then $C>c>0$.

Recall that a matrix $G \in \mathbb{R}^{n \times n}$ is said to be copositive on $K$ if

$$
\langle G x, x\rangle \geq 0 \quad \forall x \in K .
$$

A matrix $G \in \mathbb{R}^{n \times n}$ is said to be strictly copositive on $K$ if

$$
\langle G x, x\rangle>0 \quad \forall x \in K \backslash\{0\} .
$$

These classes of matrices play an important role in complementarity theory (see e.g., [21]). The set of copositive matrices contains that of positive-semidefinite (PSD) matrices [21, p. 174 ]. Indeed, a PSD matrix is necessarily copositive on any set $K$. However it is easy to construct a matrix that is copositive on a certain set $K$, but which is not PSD.

Condition (3) of Definitions 4 and 5 correspond to (3) in Theorems 2 and 3. Let us here denote by $\mathcal{P}_{K}$ (resp. $\mathcal{P}_{K}^{+}$) the set of copositive (resp. strictly copositive) matrices on $K$. Let us also denote by $\mathcal{P}_{K}^{++}$the set of matrices satisfying condition (1) of Definition 4, that is

$$
\mathcal{P}_{K}^{++}=\left\{B \in \mathbb{R}^{n \times n}: \inf _{x \in K \backslash\{0\}} \frac{\langle B x, x\rangle}{\|x\|^{2}}>0\right\} .
$$

It is clear that

$$
\begin{gathered}
\mathcal{P}_{K}^{++} \subset \mathcal{P}_{K}^{+} \subset \mathcal{P}_{K} . \\
K_{1} \subset K_{2} \Rightarrow \mathcal{P}_{K_{2}}^{++} \subset \mathcal{P}_{K_{1}}^{++} .
\end{gathered}
$$

Proposition 1: If $K$ is a cone, then

$$
\mathcal{P}_{K}^{++}=\mathcal{P}_{K}^{+} .
$$

Proof: We know that $\mathcal{P}_{K}^{++} \subset \mathcal{P}_{K}^{+}$. It suffices to check that $\mathcal{P}_{K}^{+} \subset \mathcal{P}_{K}^{++}$. Let $B \in \mathcal{P}_{K}^{+}$, that is $\langle B x, x\rangle>0, \forall x \in K \backslash\{0\}$ and let us verify that there exists $c>0$ such that $\langle B x, x\rangle \geq$ $c\|x\|^{2}, \forall x \in K \backslash\{0\}$. If we suppose the contrary, then we can find a sequence $\left\{x_{n}\right\} \subset K \backslash\{0\}$ such that $\left\langle B x_{n}, x_{n}\right\rangle<$ $(1 / n)\left\|x_{n}\right\|^{2}$. Let $z_{n}:=x_{n} /\left\|x_{n}\right\|$. We have $\left\|z_{n}\right\|=1$ and thus there exists a subsequence $\left\{z_{n_{k}}\right\}$ such that $z_{n_{k}} \rightarrow z,\|z\|=1$ and $\langle B z, z\rangle=\lim _{n_{k} \rightarrow \infty}\left\langle B z_{n_{k}}, z_{n_{k}}\right\rangle \leq 0$. This contradicts the strict copositivity of $B$.

Remark 4: Let $A \in \mathbb{R}^{n \times n}$ be a positive strictly-stable matrix, i.e.,

$$
\operatorname{re}(\lambda)>0 \quad \forall \lambda \in \sigma(A) .
$$


Then, there exists a matrix $G \in \mathbb{R}^{n \times n}$ satisfying conditions (1) and (2) of Definition 5. Indeed, let $H \in \mathbb{R}^{n \times n}$ be any positive-definite matrix. From Lyapunov's theorem (see [25]) there exists a positive-definite matrix $G \in \mathbb{R}^{n \times n}$ satisfying the Lyapunov equation $G A+A^{T} G=H$. Thus, $\left\langle A x,\left[G+G^{T}\right] x\right\rangle=$ $\langle H x, x\rangle>0, \forall x \in \mathbb{R}^{n} \backslash\{0\}$.

Remark 5: A first step in finding a matrix $G$ satisfying condition (2) of Definition 4 (resp. Definition 5 ) may consist to deal with the Lyapunov equation $G A+A^{T} G=H$ and by taking $H$ as a matrix of $\mathcal{P}_{K}$ (resp. $\mathcal{P}_{K}^{++}$). The Lyapunov equation can be written as

$$
\left(A^{T} \otimes I+I \otimes A^{T}\right) g=h
$$

where $g$ and $h$ denotes the column $n^{2}$ vectors formed from the rows of $G$ and $H$, respectively, taken in order. Note also that by choosing $G$ and $H$ symmetric then, redundant equations and variables can be removed to give a system of order $(1 / 2) n(n+$ 1).

Let us now denote by $\mathcal{L}_{K}$ the set of Lyapunov positive stable matrices on $K$ and by $\mathcal{L}_{K}^{++}$the set of Lyapunov positive strictly stable matrices on $K$. We see that

$$
\begin{aligned}
\mathcal{L}_{K}= & \left\{A \in \mathbb{R}^{n \times n}: \exists G \in \mathcal{P}_{K}^{++}\right. \text {such that } \\
& \left.\left(I-\left[G+G^{T}\right]\right)(\partial K) \subset K \text { and } G A+A^{T} G \in \mathcal{P}_{K}\right\}
\end{aligned}
$$

and

$$
\begin{aligned}
\mathcal{L}_{K}^{++}= & \left\{A \in \mathbb{R}^{n \times n}: \exists G \in \mathcal{P}_{K}^{++}\right. \text {such that } \\
& \left.\left(I-\left[G+G^{T}\right]\right)(\partial K) \subset K \text { and } G A+A^{T} G \in \mathcal{P}_{K}^{++}\right\} .
\end{aligned}
$$

Let us note that $G$ needs not be symmetric.

Definition 6: A matrix $A \in \mathbb{R}^{n \times n}$ is said to be Lyapunov unstable on $K$ if there exists a matrix $G \in \mathbb{R}^{n \times n}$ and a constant $\alpha>0$ such that

1) $G \in \mathcal{P}_{K}^{+}$

2) $\left\langle A x,\left[G+G^{T}\right] x\right\rangle \leq-\alpha\langle G x, x\rangle, \forall x \in K$;

3) $x \in \partial K \Rightarrow\left[G+\bar{G}^{T}\right] x \in K_{\infty}$.

The set of Lyapunov unstable matrices on $K$ will be denoted by $\mathcal{L}_{K}^{-}$. We have

$$
\begin{aligned}
& \mathcal{L}_{K}^{-}=\left\{A \in \mathbb{R}^{n \times n}: \exists G \in \mathcal{P}_{K}^{+} \text {and } \alpha>0\right. \text { such that } \\
& \left.-\left[\left(G+G^{T}\right) A+\alpha G\right] \in \mathcal{P}_{K} \text { and }\left[G+G^{T}\right](\partial K) \subset K_{\infty}\right\} .
\end{aligned}
$$

Remark 6: If

$$
-\infty<c:=\sup _{x \in K \backslash\{0\}} \frac{\left\langle A x,\left[G+G^{T}\right] x\right\rangle}{\|x\|^{2}}<0
$$

then (2) of Definition 6 holds with $\alpha=|c| /\|G\|$. Note that if $c:=-\infty$ then necessarily $K=\{0\}$ and condition (2) is, in this case, trivial.

Various classes of stability and instability matrices will be given later in this paper. According to the comments at the end of the foregoing section, there may exist matrices which satisfy none of the conditions of Definitions 4-6, and nevertheless are stable or unstable. Two examples are given in Section VI-D, Example 3.

\section{STABILITY OF LiNEAR EVOLUTION VARIATIONAL INEQUALITIES}

Let $K \subset \mathbb{R}^{n}$ be a closed convex set such that $0 \in K$. We consider Problem $P\left(t_{0}, x_{0}\right)$ with $F \equiv 0$, i.e.,: Find $x \in$ $C^{0}\left(\left[t_{0}, \infty\right) ; \mathbb{R}^{n}\right)$ such that $(d x / d t) \in L_{\mathrm{loc}}^{\infty}\left(t_{0},+\infty ; \mathbb{R}^{n}\right)$ and

$$
\begin{aligned}
\left\langle\frac{d x}{d t}(t)+A x(t), v-x(t)\right\rangle & \geq 0 \\
\forall v & \in K, \text { a.e. } t \geq t_{0} \\
x(t) & \in K, \quad t \geq t_{0} \\
x\left(t_{0}\right) & =x_{0}
\end{aligned}
$$

Theorem 5: Let $K \subset \mathbb{R}^{n}$ be a set satisfying hypothesis $\left(H_{1}\right)-\left(H_{3}\right)$.

- If $A \in \mathcal{L}_{K}$, then the trivial solution of (12) and (13) is stable.

- If $A \in \mathcal{L}_{K}^{++}$, then the trivial solution of (12) and (13) is asymptotically stable.

- Suppose here in addition that $K \backslash\{0\} \neq \emptyset$. If $A \in \mathcal{L}_{K}^{-}$, then the trivial solution of (12) and (13) is unstable.

Proof:

i) $A \in \mathcal{L}_{K}$ and thus there exists a matrix $G \in \mathbb{R}^{n \times n}$ such that

$$
\begin{aligned}
\inf _{x \in K \backslash\{0\}} \frac{\langle G x, x\rangle}{\|x\|^{2}}>0 \\
\left\langle A x,\left[G+G^{T}\right] x\right\rangle \geq 0 \quad \forall x \in K
\end{aligned}
$$

and

$$
x \in \partial K \Rightarrow\left[I-\left[G+G^{T}\right]\right] x \in K .
$$

Let $V \in C^{1}\left(\mathbb{R}^{n} ; \mathbb{R}\right)$ be defined by

$$
V(x)=\frac{1}{2}\left\langle\left[G+G^{T}\right] x, x\right\rangle .
$$

Then, $V^{\prime}(x)=\left[G+G^{T}\right] x$ and we see that all the assumptions of Theorem 2 are satisfied. Indeed, (15) ensures the existence of a constant $k>0$ (see Remark 3) such that

$$
V(x) \geq k\|x\|^{2} \quad \forall x \in K .
$$

It is clear that $V(0)=0$. Finally, from (16) and (17), we deduce that

$$
\left\langle A x, V^{\prime}(x)\right\rangle \geq 0 \quad \forall x \in K
$$

and

$$
x \in \partial K \Rightarrow x-V^{\prime}(x) \in K .
$$

The conclusion follows from Theorem 2 . 
ii) $A \in \mathcal{L}_{K}^{++}$and thus there exists a matrix $G \in \mathbb{R}^{n \times n}$ satisfying (15), (17), and

$$
\inf _{x \in K \backslash\{0\}} \frac{\left\langle A x,\left[G+G^{T}\right] x\right\rangle}{\|x\|^{2}}>0 .
$$

We define $V \in C^{1}\left(\mathbb{R}^{n} ; \mathbb{R}\right)$ as in (18) and we verify as in part i) that assumptions 1), 2), and 3 ) of Theorem 3 are satisfied. Moreover, from (19), we deduce the existence of a constant $c>0$ such that

$$
\left\langle A x,\left[G+G^{T}\right] x\right\rangle \geq c\|x\|^{2} \quad \forall x \in K .
$$

It results that

$$
\begin{aligned}
\left\langle A x,\left[G+G^{T}\right] x\right\rangle & \geq \frac{c}{\left\|G+G^{T}\right\|}\left\langle\left[G+G^{T}\right] x, x\right\rangle \\
\forall x & \in K .
\end{aligned}
$$

Thus

$$
\left\langle A x,\left[G+G^{T}\right] x\right\rangle \geq \lambda V(x) \quad \forall x \in K
$$

with $\lambda:=2 c /\left\|G+G^{T}\right\|$. This yields Assumption (4) of Theorem 3. The conclusion follows from Theorem 3 .

iii) If $A \in \mathcal{L}_{K}^{-}$then there exists a matrix $G \in \mathbb{R}^{n \times n}$ and $\alpha>0$ such that

$$
\begin{gathered}
\langle G x, x\rangle>0 \quad \forall x \in K \backslash\{0\} \\
x \in \partial K \Rightarrow\left[G+G^{T}\right] x \in K_{\infty}
\end{gathered}
$$

and

$\left(A x,\left[G+G^{T}\right] x\right) \leq-\alpha\langle G x, x\rangle \quad \forall x \in K$.

Let $V \in C^{1}\left(\mathbb{R}^{n} ; \mathbb{R}\right)$ be defined by

$$
V(x)=\frac{1}{2}\left\langle\left[G+G^{T}\right] x, x\right\rangle .
$$

We see that all the conditions of Theorem 4 are satisfied. It is clear that (1) is satisfied with $b(t)=(1 / 2) \| G+$ $G^{T} \| t^{2}$. Condition (20) ensures that (2) is satisfied. Condition (21) yields (3). Finally, from (22), we deduce that

$$
\left\langle A x, V^{\prime}(x)\right\rangle \leq-\alpha V(x) \quad \forall x \in K
$$

so that assumption (4) holds too. The conclusion follows from Theorem 4.

\section{NONLINEAR PERTURbations OF Linear VARIATIONAL INEQUALITIES}

Let $K \subset \mathbb{R}^{n}$ be a closed convex set such that $0 \in K$. Let us now consider problem $P\left(t_{0}, x_{0}\right)$ : Find $x \in C^{0}\left(\left[t_{0}, \infty\right) ; \mathbb{R}^{n}\right)$ such that $(d x / d t) \in L_{\mathrm{loc}}^{\infty}\left(t_{0},+\infty ; \mathbb{R}^{n}\right)$ and

$$
\begin{aligned}
\left\langle\frac{d x}{d t}(t)+A x(t)+F(x(t)), v-x(t)\right\rangle & \geq 0 \\
\forall v \in K, \text { a.e. } t & \geq t_{0} \\
x(t) \in K, \quad t & \geq t_{0} \\
x\left(t_{0}\right) & =x_{0} .
\end{aligned}
$$

Theorem 6 : Let $K \subset \mathbb{R}^{n}$ be a set satisfying hypothesis $\left(H_{1}\right)-\left(H_{3}\right)$. Let $F: \mathbb{R}^{n} \rightarrow \mathbb{R}^{n}$ be given by

$$
F=F_{1}+\Phi^{\prime}
$$

where $F_{1}$ is Lipschitz continuous and $\Phi \in C^{1}\left(\mathbb{R}^{n} ; \mathbb{R}\right)$ is convex. Suppose also that

$$
\lim _{\|x\| \rightarrow 0} \frac{\|F(x)\|}{\|x\|}=0 .
$$

If $A \in \mathcal{L}_{K}^{++}$then the trivial solution of (23) and (24) is asymptotically stable.

Proof: There exists a matrix $G \in \mathbb{R}^{n \times n}$ such that

$$
\begin{array}{r}
\inf _{x \in K \backslash\{0\}} \frac{\langle G x, x\rangle}{\|x\|^{2}}>0 \\
\inf _{x \in K \backslash\{0\}} \frac{\left\langle A x,\left[G+G^{T}\right] x\right\rangle}{\|x\|^{2}}>0
\end{array}
$$

and

$$
x \in \partial K \Longrightarrow x-\left[G+G^{T}\right] x \in K .
$$

Our aim is to verify that all conditions of Theorem 3 are satisfied with $V \in C^{1}\left(\mathbb{R}^{n} ; \mathbb{R}\right)$ defined by

$$
V(x)=\frac{1}{2}\left\langle\left[G+G^{T}\right] x, x\right\rangle
$$

From (27), we see that there exists a constant $c_{1}>0$ such that

$$
V(x)=\langle G x, x\rangle \geq c_{1}\|x\|^{2} \quad \forall x \in K .
$$

This yields Assumption 1) of Theorem 3. It is clear that $V(0)=$ 0 so that Assumption 2) of Theorem 3 is also satisfied. Here, $V^{\prime}(x)=\left[G+G^{T}\right] x$ and (29) yields Assumption 3) of Theorem 3 . Finally, from (28), we obtain that

$$
\left\langle A x, V^{\prime}(x)\right\rangle \geq c_{2}\|x\|^{2} \quad \forall x \in K
$$

for some constant $c_{2}>0$. On the other hand, because of (26) there exists a constant $\sigma>0$ such that

$$
\|x\| \leq \sigma \Rightarrow\|F(x)\| \leq \frac{c_{2}}{\left\|G+G^{T}\right\|}\|x\| .
$$

Thus, if $\|x\| \leq \sigma$ then

$$
\begin{aligned}
\left\langle F(x), V^{\prime}(x)\right\rangle & =\frac{1}{2}\left\langle F(x),\left[G+G^{T}\right] x\right\rangle \\
& \geq-\frac{1}{2}\left\|G+G^{T}\right\|\|F(x)\|\|x\| \\
& \geq-\frac{1}{2} c_{2}\|x\|^{2} .
\end{aligned}
$$

It results that

$$
\left\langle A x+F(x), V^{\prime}(x)\right\rangle \geq \frac{c_{2}}{2}\|x\|^{2} \quad \forall x \in K,\|x\| \leq \sigma
$$

and, thus, Assumption 4) of Theorem 3 holds with $\lambda=c_{2} /\left\|G+G^{T}\right\|$.

Theorem 7: Let $K \subset \mathbb{R}^{n}$ be a set satisfying hypothesis $\left(H_{1}\right)-\left(H_{3}\right)$. Let $F: \mathbb{R}^{n} \rightarrow \mathbb{R}^{n}$ be given by

$$
F=F_{1}+\Phi^{\prime}
$$


where $F_{1}$ is Lipschitz continuous and $\Phi \in C^{1}\left(\mathbb{R}^{n} ; \mathbb{R}\right)$ is convex. Suppose also that

$$
-\Phi^{\prime}(0) \in N_{K}(0)
$$

and

$$
\lim _{\|x\| \rightarrow 0} \frac{\left\|F_{1}(x)\right\|}{\|x\|}=0 .
$$

If $A \in \mathcal{P}_{K}^{++}$, then the trivial solution of (23) and (24) is asymptotically stable.

Proof: We first note that (30) and (31) ensure that $-F(0) \in N_{K}(0)$. Let us now define $V \in C^{1}\left(\mathbb{R}^{n} ; \mathbb{R}\right)$ by the formula

$$
V(x)=\frac{1}{2}\|x\|^{2} .
$$

We see that all the assumptions of Theorem 3 are satisfied. Indeed, assumptions (1), (2) and (3) are clearly satisfied. Moreover, $A \in \mathcal{P}_{K}^{++}$and thus there exists a constant $c>0$ such that

$$
\langle A x, x\rangle \geq c\|x\|^{2} \quad \forall x \in K .
$$

On the other hand, $\Phi^{\prime}$ is monotone and, thus

$$
\left\langle\Phi^{\prime}(x), x\right\rangle \geq\left\langle\Phi^{\prime}(0), x\right\rangle \quad \forall x \in \mathbb{R}^{n} .
$$

From (30), it results that

$$
\left\langle\Phi^{\prime}(x), x\right\rangle \geq 0 \quad \forall x \in K .
$$

Finally, (31) ensures the existence of $\sigma>0$ such that

$$
\|x\| \leq \sigma \Rightarrow\left\|F_{1}(x)\right\| \leq \frac{c}{2}\|x\| .
$$

It results that if $x \in K$, then

$$
\begin{aligned}
\left\langle A x+F(x), V^{\prime}(x)\right\rangle & =\left\langle A x+F_{1}(x)+\Phi^{\prime}(x), x\right\rangle \\
& \geq c\|x\|^{2}-\left\|F_{1}(x)\right\|\|x\|
\end{aligned}
$$

and, thus

$$
\begin{aligned}
\left\langle A x+F_{1}(x)+\Phi^{\prime}(x), V^{\prime}(x)\right\rangle & \geq \frac{c}{2}\|x\|^{2} \\
\forall x \in K, \quad\|x\| & \leq \sigma .
\end{aligned}
$$

That means that assumption (4) of Theorem 3 is satisfied with $\lambda=c$. The conclusion follows from Theorem 3 .

\section{STABILITY AND INSTABILITY CRITERIA AND EXAMPLES}

\section{A. Stability Matrices}

Let us first give important classes of matrices satisfying the conditions of definition 4 and definition 5. Let $K \subset \mathbb{R}^{n}$ be a set satisfying hypothesis $\left(H_{1}\right)-\left(H_{3}\right)$.

Proposition 2: Suppose that $A \in \mathcal{P}_{K}$, i.e.,

$$
\langle A x, x\rangle \geq 0 \quad \forall x \in K .
$$

Then, $A \in \mathcal{L}_{K}$.

Proof: Let $G=(1 / 2) I$. Conditions (1) and (2) of Definition 4 are clearly satisfied. Moreover, $\left(I-\left[G+G^{T}\right]\right) x=0 \in$ $K$.
Proposition 3: Suppose that $A \in \mathcal{P}_{K}^{++}$, i.e.,

$$
\inf _{x \in K \backslash\{0\}} \frac{\langle A x, x\rangle}{\|x\|^{2}}>0 .
$$

Then, $A \in \mathcal{L}_{K}^{++}$.

Proof: As in the proof of Proposition 2, we see that the choice $G=(1 / 2) I$ is convenient.

Remark 7: From Propositions 2 and 3, we deduce that

$$
\mathcal{P}_{K} \subset \mathcal{L}_{K} \quad \mathcal{P}_{K}^{++} \subset \mathcal{L}_{K}^{++} .
$$

We see now that, with some additional conditions imposed on the set $K$, it is possible to consider larger classes of matrices.

Proposition 4: Suppose that $K$ is a cone such that

$$
x \in \partial K \Rightarrow x_{i} \bar{e}_{i} \in K, \quad(i=1, \ldots, n)
$$

where $\bar{e}_{i}$ denotes the $i$ th canonical vector of $\mathbb{R}^{n}$. If there exists a positive-diagonal matrix $D$ such that

$$
D A \in \mathcal{P}_{K}
$$

then $A \in \mathcal{L}_{K}$.

Proof: We set $G:=(1 / 2 \alpha) D$, where $\alpha:=$ $\max _{1 \leq i \leq n}\left\{d_{i i}\right\}$. The matrix $G$ is symmetric and positive definite. Moreover, $\left\langle A x,\left[G+G^{T}\right] x\right\rangle=(1 / \alpha)\langle D A x, x\rangle$ and thus $\left\langle A x,\left[G+G^{T}\right] x\right\rangle \geq 0, \forall x \in K$. Finally, $\left(I-\left[G+G^{T}\right]\right) x=$ $\operatorname{diag}_{1 \leq i \leq n}\left\{\left(1-\left(d_{i i} / \alpha\right)\right) x_{i}\right\}=\sum_{i=1}^{n}\left(1-\left(d_{i i} / \alpha\right)\right) x_{i} \bar{e}_{i}$. Then, using the assumptions on $K$, we see that for $x \in \partial K$ we have $\left(I-\left[G+G^{T}\right]\right) x \in K$.

Proposition 5: Suppose that $K$ is a cone such that

$$
x \in \partial K \Rightarrow x_{i} \bar{e}_{i} \in K, \quad(i=1, \ldots, n) .
$$

If there exists a positive-diagonal matrix $D$ such that

$$
D A \in \mathcal{P}_{K}^{++}
$$

then $A \in \mathcal{L}_{K}^{++}$.

Proof: As in Proposition 4, we set $G:=(1 / 2 \alpha) D$ with $\alpha:=\max _{1 \leq i \leq n}\left\{d_{i i}\right\}$. Here, $\left\langle A x,\left[G+G^{T}\right] x\right\rangle=(1 / \alpha)\langle D A x, x\rangle$ and, thus, $\inf _{x \in K \backslash\{0\}}\left(\left\langle A x,\left[G+G^{T}\right] x\right\rangle /\|x\|^{2}\right)>0$. The proof is achieved as in Proposition 4.

Remark 8: If $A$ is a nonsingular $M$-matrix, i.e., $A=s I-P$; $s>\rho(P), P$ is such that $p_{i j} \geq 0$, for all $i, j \in\{1, \ldots, n\}$, then there exists a positive-diagonal matrix $D$ such that

$$
H:=D A+A^{T} D
$$

is positive definite (see, e.g., [13] ). It results that $\langle A x, D x\rangle=$ $(1 / 2)\left\langle A x,\left[D+D^{T}\right] x\right\rangle=(1 / 2)\left\langle\left[A^{T} D+D A\right] x, x\right\rangle$ $=(1 / 2)\langle H x, x\rangle \geq(1 / 2) \lambda_{\min }\left(H+H^{T} / 2\right)\|x\|^{2}$. Thus $\inf _{x \in K \backslash\{0\}}\left(\langle D A x, x\rangle /\|x\|^{2}\right)>0$ and the condition required in Proposition 5 on the matrix $A$ is satisfied.

Proposition 6: Suppose that $K$ satisfies the property

$$
x \in \partial K \Rightarrow x_{i} \bar{e}_{i} \in K \quad(i=1, \ldots, n) .
$$

If there exists a positive-diagonal matrix $D$ such that

1) $(\operatorname{tr}(D) / n-1) \geq \max _{1 \leq i \leq n}\left\{d_{i i}\right\}$;

2) $D A \in \mathcal{P}_{K}$. 
Then, $A \in \mathcal{L}_{K}$.

Proof: Let $\alpha:=(\operatorname{tr}(D) / n-1)$. We set $G:=(1 / 2 \alpha) D$ It is clear that $G \in P_{K}^{++}$. Moreover, $\left\langle A x,\left[G+G^{T}\right] x\right\rangle=$ $(1 / \alpha)\langle D A x, x\rangle \geq 0$. Finally, $\left(I-\left[G+G^{T}\right]\right) x=\sum_{i=1}^{n}(1-$ $\left(d_{i i} / \alpha\right) x_{i} \bar{e}_{i}$. If $x \in \partial K$, then $x_{i} \bar{e}_{i} \in K$. Moreover, $0 \leq$ $\left(d_{i i} / \alpha\right) \leq 1$ since (1) holds and $\sum_{i=1}^{n}\left(1-\left(d_{i i} / \alpha\right)\right)=n-$ $(\operatorname{tr} D / \alpha)=1$. It results that, for $x \in \partial K$, we have $(I-[G+$ $\left.\left.G^{T}\right]\right) x \in K$.

Proposition 7: Suppose that $K$ satisfies the property

$$
x \in \partial K \Rightarrow x_{i} \bar{e}_{i} \in K \quad(i=1, \ldots, n) .
$$

If there exists a positive-diagonal matrix $D$ such that

1) $(\operatorname{tr}(D) / n-1) \geq \max _{1<i<n}\left\{d_{i i}\right\}$;

2) $D A \in \mathcal{P}_{K}^{++}$.

Then, $A \in \mathcal{L}_{K}^{++}$.

Proof: With the choice of $G$ as in the proof of Proposition 6, we see that (1) and (3) of Definition 5 hold. Moreover, as above, we check that $\left\langle A x,\left[G+G^{T}\right] x\right\rangle=(1 / \alpha)\langle D A x, x\rangle$ and thus using assumption (2), we obtain $\inf _{x \in K \backslash\{0\}}\left(\left\langle A x,\left[G+G^{T}\right] x\right\rangle /\|x\|^{2}\right)>0$.

Proposition 8: Suppose that $K$ is symmetric. If there exists $\lambda \geq 0$ such that

$$
\left(I+\lambda P_{K_{\infty}}\right) A \in \mathcal{P}_{K}
$$

then $A \in \mathcal{L}_{K}$.

Proof: We know that

$$
\left\langle P_{K_{\infty}} x-x, y-P_{K_{\infty}} x\right\rangle \geq 0 \quad \forall y \in K_{\infty}
$$

and thus letting $y=0$ we get

$$
\left\langle P_{K_{\infty}} x, x\right\rangle \geq\left\|P_{K_{\infty}} x\right\|^{2} .
$$

Let

$$
G=\frac{1}{2}\left[I+\lambda P_{K_{\infty}}\right]
$$

Then

$$
\langle G x, x\rangle \geq \frac{1}{2}\|x\|^{2}+\frac{\lambda}{2}\left\|P_{K_{\infty}}\right\|^{2}
$$

and $G \in \mathcal{P}_{K}^{++}$. We have also $\left\langle A x,\left[G+G^{T}\right] x\right\rangle=$ $\langle A x, x\rangle+\lambda\left\langle A x, P_{K_{\infty}} x\right\rangle=\langle A x, x\rangle+\lambda\left\langle P_{K_{\infty}} A x, x\right\rangle=$ $\left\langle\left[I+\lambda P_{K_{\infty}}\right] A x, x\right\rangle$. Thus, by assumption $\left\langle A x,\left[G+G^{T}\right] x\right\rangle \geq$ $0, \forall x \in K$. Finally, recalling that here $-K_{\infty}=K_{\infty} \subset K$, we see that $x-\left[G+G^{T}\right] x=-\lambda P_{K_{\infty}} x \in K$.

Proposition 9: Suppose that $K$ is symmetric. If there exists $\lambda \geq 0$ such that

$$
\left(I+\lambda P_{K_{\infty}}\right) A \in \mathcal{P}_{K}^{++}
$$

then $A \in \mathcal{L}_{K}^{++}$.

Proof: We choose $G$ as in Proposition 8 and check that $\left\langle A .,\left[G+G^{T}\right].\right\rangle=\left\langle\left[I+\lambda P_{K_{\infty}}\right] A .,.\right\rangle$. By assumption, it results that $\inf _{x \in K \backslash\{0\}}\left(\left\langle A x,\left[G+G^{T}\right] x\right\rangle /\|x\|^{2}\right)>0$. The proof is achieved as in Proposition 8.

Proposition 10 : If there exists a symmetric nonsingular $M$-matrix $Q$ such that

$$
Q A \in \mathcal{P}_{\mathbf{R}_{+}^{n}}
$$

then $A \in \mathcal{L}_{\mathbb{R}_{+}^{n}}$.
Proof: There exists $P$ with $p_{i j} \geq 0, \forall i, j \in\{1, \ldots, n\}$ and $s>\rho(P)$ such that $Q=s I-P$. We set $G:=(1 / 2 s) Q$. The matrix $G$ is positive definite and, by assumption, $Q A \in$ $\mathcal{P}_{\mathbf{R}_{+}^{n}}$. Moreover, $I-\left(G+G^{T}\right)=(1 / s) P$ so that $[(I-(G+$ $\left.\left.G^{T}\right)\right]\left(\mathbb{R}_{+}^{n}\right) \subset \mathbb{R}_{+}^{n}$.

Proposition 11: If there exists a symmetric nonsingular $M$-matrix $Q$ such that

$$
Q A \in \mathcal{P}_{\mathbf{R}_{+}^{n}}^{+}
$$

then $A \in \mathcal{L}_{\mathbf{R}_{+}^{n}}^{++}$.

Proof: The proof is similar to the one of Proposition 10.

Proposition 12: Let us consider a linear state transformation $z=L x, L \in \mathbb{R}^{n \times n}$ full-rank, and $L L^{T}=I$. Then $A \in \mathcal{L}_{K}$ (respectively, $A \in \mathcal{L}_{K}^{++}$) if and only if $\bar{A}=L A L^{T} \in \mathcal{L}_{L(K)}$ (resp. $\bar{A} \in \mathcal{L}_{L(K)}^{++}$) with $G$ (see Definitions 4 and 5) transformed to $\bar{G}=L G L^{T}$.

Proof: The set $L(K)=\left\{z \in \mathbb{R}^{n}: x \in K\right.$ and $\left.z=L x\right\}$ is convex and closed since $K_{\infty} \cap \operatorname{Ker}(L)=\{0\}$ [19, p. 71$]$. Since $\{z \in L(K)\} \Leftrightarrow\{x \in K\}$, it follows that $\left\{G \in \mathcal{P}_{K}\right\} \Leftrightarrow$ $\left\{\bar{G} \in \mathcal{P}_{L(K)}\right\}$. Moreover, for $x \in \partial K \Leftrightarrow z \in \partial L(K)$ one has $\left(I-\left[G+G^{T}\right]\right) x \in K$ if and only if $\left(I-\left[\bar{G}+\bar{G}^{T}\right]\right) z \in L(K)$.

\section{B. Examples}

Let us here illustrate the previous results with some simple examples.

Example 1:

i) Let $K:=\mathbb{R}_{+} \times \mathbb{R}_{+}$and

$$
A=\left(\begin{array}{ll}
1 & 2 \\
1 & 1
\end{array}\right) .
$$

It is clear that $A \in \mathcal{P}_{k}$ and from Proposition 2 we obtain that $A \in \mathcal{L}_{k}$.

ii) Let $K=\left(\mathbb{R}_{+}\right)^{n}$ and $A \in \mathbb{R}^{n \times N}$ such that $A \geq 0$. Then, clearly

$$
\langle x, A x\rangle \geq 0, \forall x \in K
$$

and, thus, $A \in \mathcal{P}_{K}$. Proposition 2 ensures that $A \in \mathcal{L}_{K}$.

iii) Let $K:=\left\{x \in \mathbb{R}^{2}: x_{1} \geq 0, x_{2}=0\right\}$ and

$$
A=\left(\begin{array}{cc}
3 & 0 \\
1 & -4
\end{array}\right) \text {. }
$$

Here, $x \in K \rightarrow\langle A x, x\rangle=3 x_{1}^{2}$ and $\inf _{x \in K \backslash\{0\}}\langle A x, x\rangle /|x|^{2}=3$. It results that $A \in \mathcal{P}_{K}^{++}$. Proposition 3 ensures that $A \in \mathcal{L}_{k}^{++}$.

iv) Let $K=\mathbb{R}_{+} \times \mathbb{R}_{+}$and

$$
A=\left(\begin{array}{cc}
1 & -10 \\
0 & 2
\end{array}\right) \text {. }
$$

The matrix $A$ is a nonsingular $M$-matrix. Moreover, $K$ is a cone and if $x \in K$ then $x_{i} \bar{e}_{i} \in K(i=1, \ldots, n)$. Using Proposition 5 and Remark 8, we obtain that $A \in$ $\mathcal{L}_{k}^{++}$.

v) Let $K=\mathbb{R}_{+} \times \mathbb{R}_{+} \times \mathbb{R}_{+}$and

$$
A=\left(\begin{array}{ccc}
1 & -4 & 1 \\
1 & 1 & 0 \\
0 & 1 & 1
\end{array}\right) \text {. }
$$


Setting $D=\operatorname{diag}\{1 / 5,1,1 / 5\}$, we check easily that $\langle A x, D x\rangle>0, \forall X \in K \backslash\{0\}$. Using Proposition 5, we get $A \in \mathcal{L}_{K}^{++}$.

vi) Let $K=\left\{x \in \mathbb{R}_{+} \times \mathbb{R}_{+} \times \mathbb{R}_{+}:\|x\| \leq 1\right\}$ and

$$
A=\left(\begin{array}{ccc}
1 & -4 & 0 \\
1 & 1 & 0 \\
0 & 0 & 0
\end{array}\right) .
$$

It is clear that if $x \in \partial K$ then $x_{i} \bar{e}_{i} \in K(i=$ $1, \ldots, N)$. Set $D=\operatorname{diag}\{1,5,5\}$. Here $\operatorname{tr}\{D\} \geq$ $(n-1) \max _{1 \leq I \leq 3}\left\{d_{i i}\right\}$ and, thus, condition 1) of Proposition 6 holds. Moreover

$$
D A=\left(\begin{array}{ccc}
1 & -4 & 0 \\
5 & 5 & 0 \\
0 & 0 & 0
\end{array}\right)
$$

and thus $\langle D A x, x\rangle=x_{1}^{2}+5 x_{2}^{2}+x_{1} x_{2} \geq 0, \forall x \in K$. All the conditions of Proposition 6 hold and, thus, $A \in$ $\mathcal{L}_{K}$.

vii) Let $K$ be defined as in example vi) and set

$$
A=\left(\begin{array}{ccc}
1 & -4 & 0 \\
1 & 1 & 0 \\
0 & 0 & 1
\end{array}\right) .
$$

Let $D$ be defined as in example vi). we have

$$
D A=\left(\begin{array}{ccc}
1 & -4 & 0 \\
5 & 5 & 0 \\
0 & 0 & 5
\end{array}\right)
$$

and, thus, $\langle D A x, x\rangle=x_{1}^{2}+5 x_{2}^{2}+5 x_{3}^{2}+x_{1} x_{2}$. It results that $\inf _{x \in K \backslash\{0\}}\left(\langle D A x, x\rangle /\|x\|^{2}\right) \geq 1$. From Proposition 7, we deduce that $A \in \mathcal{L}_{K}^{++}$.

viii) Let $K=\left\{x \in \mathbb{R}^{3}:-1 \leq x_{3} \leq 1\right\}$ and

$$
A=\left(\begin{array}{ccc}
0 & 0 & 0 \\
0 & 1 & 1 \\
0 & -2 & 2
\end{array}\right) .
$$

The set $K$ is symmetric and $K_{\infty}=\left\{x \in \mathbb{R}^{3}: x_{3}=0\right\}$. Then

$$
P_{K_{\infty}}=\left(\begin{array}{lll}
1 & 0 & 0 \\
0 & 1 & 0 \\
0 & 0 & 0
\end{array}\right) .
$$

Setting $\lambda=1$, we see that $I+\lambda P_{K_{\infty}}=\operatorname{diag}\{2,2,1\}$ and $\left\langle\left[I+\lambda P_{K_{\infty}}\right] A x, x\right\rangle=2 x_{2}^{2}+2 x_{3}^{2} \geq 0, \forall x \in K$. Using Proposition 8, we see that $A \in \mathcal{L}_{K}$.

ix) Let $K$ be defined as in example viii) and set

$$
A=\left(\begin{array}{ccc}
1 & 0 & 0 \\
0 & 1 & 1 \\
0 & -4 & 1
\end{array}\right) .
$$

Setting $\lambda=3$, we see that $I+\lambda P_{K_{\infty}}=\operatorname{diag}\{4,4,1\}$ and $\left(\left[I+\lambda P_{K_{\infty}}\right] A x, x\right)=4 x_{1}^{2}+4 x_{2}^{2}+x_{3}^{2}$. Thus, $\left[I+\lambda P_{K_{\infty}}\right] A \in \mathcal{P}_{K}^{++}$and Proposition 9 ensures that $A \in \mathcal{L}_{K}^{++}$. x) Let $K=\left\{x \in \mathbb{R}^{2}: x_{1}-1 \leq x_{2} \leq x_{1}+1\right\}$ and

$$
A=\left(\begin{array}{cc}
2 & 2 \\
-2 & 0
\end{array}\right) \text {. }
$$

The set $K$ is symmetric and $K_{\infty}=\left\{x \in \mathbb{R}^{2}: x_{2}=\right.$ $\left.x_{1}\right\}$. We have

$$
P_{K_{\infty}}=\left(\begin{array}{cc}
\frac{1}{2} & \frac{1}{2} \\
\frac{1}{2} & \frac{1}{2}
\end{array}\right)
$$

and setting $\lambda=2$, we see that

$$
\left[I+\lambda P_{K_{\infty}}\right] A=\left(\begin{array}{cc}
2 & 4 \\
-2 & 2
\end{array}\right) .
$$

Thus, $\left\langle\left[I+\lambda P_{K_{\infty}}\right] A x, x\right\rangle$ is positive definite and $\left.\inf _{x \in K \backslash\{0\}}\left(\left[I+\lambda P_{K_{\infty}}\right] a x, x\right\rangle /|x|^{2}\right)>0$. Using Proposition 9, we obtain that $A \in \mathcal{L}_{K}^{++}$.

xi) If $A=\operatorname{diag}\{-a, b\}$ with $a>0, b>0$, then $A \in \mathcal{P}_{K}$ for all closed convex sets $K \subseteq\left\{-\sqrt{a / b} x_{1} \leq x_{2} \leq\right.$ xii) If $\left.\sqrt{a / b} x_{1}\right\}$

$$
A=\left(\begin{array}{cc}
0 & -b \\
c & 0
\end{array}\right)
$$

$b>0, c>0$, then (1) $A \in \mathcal{P}_{\mathbf{R}_{+}^{2}} \cap \mathcal{P}_{\mathbf{R}_{-}^{2}}$ if $-b+c>0$,

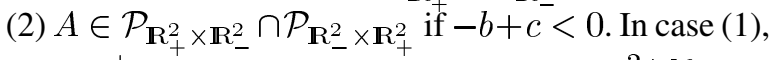
$A \in \mathcal{P}_{K}^{+}$for all closed convex sets $K \subset \mathbb{R}_{+}^{2} \backslash\left[\left\{x_{1}=\right.\right.$ $\left.0\} \cup\left\{x_{2}=0\right\}\right]$ and for all closed convex sets $K \subset$ $\mathbb{R}_{-}^{2} \backslash\left[\left\{x_{1}=0\right\} \cup\left\{x_{2}=0\right\}\right]$. In case 2$)$, this holds for the quadrants $\mathbb{R}^{-} \times \mathbb{R}^{+} \backslash\left\{\left\{x_{1}=0\right\} \cup\left\{x_{2}=0\right\}\right\}$ and $\mathbb{R}^{+} \times \mathbb{R}^{-} \backslash\left\{\left\{x_{1}=0\right\} \cup\left\{x_{2}=0\right\}\right\}$.

\section{Instability Matrices}

Let us now provide some criteria to characterize instability matrices.

Proposition 13: Suppose that $K$ is a cone. If $-A \in \mathcal{P}_{K}^{+}$, then $A \in \mathcal{L}_{K}^{-}$.

Proof: We set $G=(1 / 2) I$. The set $K$ is a cone and thus there exists $c>0$ such that $\langle-A x, x\rangle \geq c\|x\|^{2}, \forall x \in K$. For $x \in K$, we have $\left\langle A x,\left[G+G^{T}\right] x\right\rangle=\langle A x, x\rangle \leq-c\|x\|^{2}=$ $-2 c\langle G x, x\rangle$. It results that (2) of Definition 6 holds with $\alpha=$ $2 c$. Moreover, $K_{\infty}=K$ and thus $x \in \partial K \subset K \Rightarrow[G+$ $\left.G^{T}\right] x=x \in K_{\infty}$.

Proposition 14: Suppose that $K$ is a cone such that

$$
x \in \partial K \Rightarrow x_{i} \bar{e}_{i} \in K(i=1, \ldots, n) .
$$

If there exists a positive-diagonal matrix $D$ such that

$$
-D A \in \mathcal{P}_{K}^{+}
$$

then $A \in \mathcal{L}_{K}^{-}$.

Proof: We set $G=(1 / 2) D$. As in the proof of Proposition 4, we check that $\left\langle A x,\left[G+G^{T}\right] x\right\rangle=\langle D A x, x\rangle$. Here, $K$ is a cone and, thus, $\mathcal{P}_{K}^{+}=\mathcal{P}_{K}^{++}$. Thus, there exists $c>0$ such that $\langle-D A x, x\rangle \geq c\|x\|^{2}, \forall x \in K$. Thus $\langle A x,[G+$ $\left.\left.G^{T}\right] x\right\rangle \leq-c\|x\|^{2} \leq(-2 c /\|D\|)\langle G x, x\rangle, \forall x \in K$. Setting $\alpha=(2 c /\|D\|)$ we see that (2) of Definition 6 holds. Moreover, 
if $x \in \partial K$ then $\left[G+G^{T}\right] x=D x=\sum_{i=1}^{n} d_{i i}\left(x_{i} \bar{e}_{i}\right) \in K_{\infty}=$ $K$.

Proposition 15: If there exist $\lambda \in \sigma(A) \cap \mathbb{R}$ and $U_{\lambda} \in$ $E_{A^{T}}(\lambda) \backslash\{0\}$ such that

1) $\lambda<0$

2) $\operatorname{ker}\left\{U_{\lambda} \otimes U_{\lambda}^{T}\right\} \cap K=\{0\}$;

3) $x \in \partial K \Rightarrow\left(U_{\lambda} \otimes U_{\lambda}^{T}\right) x \in K_{\infty}$; then $A \in \mathcal{L}_{K}^{-}$.

Proof: We first remark that

$$
\left[U_{\lambda} \otimes U_{\lambda}^{T}\right] x=\left\langle U_{\lambda}, x\right\rangle U_{\lambda}
$$

Setting $G:=U_{\lambda} \otimes U_{\lambda}^{T}$, we see that $G$ is symmetric and

$$
\langle G x, x\rangle=\left\langle U_{\lambda}, x\right\rangle^{2} \geq 0 \quad \forall x \in \mathbb{R}^{n}
$$

and, thus, $G$ is PSD. It results that

$$
\operatorname{ker}\{G\}=\left\{x \in \mathbb{R}^{n}:\langle G x, x\rangle=0\right\} .
$$

Assumption 2) ensures that

$$
\{x \in K:\langle G x, x\rangle=0\}=\{0\}
$$

and, thus

$$
\langle G x, x\rangle>0 \quad \forall x \in K \backslash\{0\} .
$$

It results that $G \in \mathcal{P}_{K}^{+}$.

We have $\left\langle A x,\left[G+G^{T}\right] x\right\rangle=2\left\langle A x, U_{\lambda}\right\rangle\left\langle U_{\lambda}, x\right\rangle=$ $2\left\langle x, A^{T} U_{\lambda}\right\rangle\left\langle U_{\lambda}, x\right\rangle=2 \lambda\left\langle U_{\lambda}, x\right\rangle^{2}=2 \lambda\left\langle\left[U_{\lambda} \otimes U_{\lambda}^{T}\right] x, x\right\rangle$. Setting $\alpha:=2|\lambda|$, we see that $\left\langle A x,\left[G+G^{T}\right] x\right\rangle=-\alpha\langle G x, x\rangle$, $\forall x \in K$. This together with assumption 3) yield the conditions of Definition 6.

Proposition 16: Suppose that there exist $\lambda, \mu \in \sigma(A) \cap \mathbb{R}$, $U_{\lambda} \in E_{A^{T}}(\lambda) \backslash\{0\}$ and $U_{\mu} \in E_{A^{T}}(\mu) \backslash\{0\}$ such that

1) $\lambda+\mu<0$

2) $\left\langle\left[U_{\lambda} \otimes U_{\mu}^{T}\right] x, x\right\rangle>0, \forall x \in K \backslash\{0\}$;

3) $x \in \partial K \stackrel{\Rightarrow}{\Rightarrow}\left(U_{\lambda} \otimes U_{\mu}^{T}+U_{\mu} \otimes U_{\lambda}^{T}\right) x \in K_{\infty}$; then $A \in \mathcal{L}_{K}^{-}$.

Proof: We first remark that

$$
\left(U_{\lambda} \otimes U_{\mu}^{T}+U_{\mu} \otimes U_{\lambda}^{T}\right) x=\left\langle U_{\mu}, x\right\rangle U_{\lambda}+\left\langle U_{\lambda}, x\right\rangle U_{\mu} .
$$

Let us set

$$
G:=U_{\lambda} \otimes U_{\mu}^{T} .
$$

Assumptions (2) and (3) yield (1) and (3) of Definition 6. We have $\left\langle A x,\left[G+G^{T}\right] x\right)=\left\langle A x,\left\langle U_{\mu}, x\right\rangle U_{\lambda}+\left\langle U_{\lambda}, x\right\rangle U_{\mu}\right)=$ $\left\langle U_{\mu}, x\right)\left\langle A x, U_{\lambda}\right\rangle+\left\langle U_{\lambda}, x\right\rangle\left\langle A x, U_{\mu}\right\rangle=\left\langle U_{\mu}, x\right\rangle\left\langle x, A^{T} U_{\lambda}\right\rangle+$ $\left\langle U_{\lambda}, x\right)\left\langle x, A^{T} U_{\mu}\right\rangle=(\lambda+\mu)\left[\left\langle U_{\lambda}, x\right\rangle\left\langle U_{\mu}, x\right\rangle\right]=(\lambda+\mu)\left\langle\left[U_{\lambda} \otimes\right.\right.$ $\left.\left.U_{\mu}^{T}\right] x, x\right\rangle$. and setting $\alpha:=|\lambda+\mu|$, we obtain that $\langle A x,[G+$ $\left.\left.G^{T}\right] x\right\rangle=-\alpha\langle G x, x\rangle, \forall x \in K$.

Proposition 17: Suppose that $K$ is a cone satisfying the properties

$$
\begin{aligned}
& \stackrel{o}{K} \neq \emptyset \\
& x \in K, y \in K \Rightarrow\langle x, y\rangle \geq 0 \\
& x \in \stackrel{\circ}{K}, y \in K,\langle x, y\rangle=0 \Rightarrow y=0 .
\end{aligned}
$$

If there exist $\lambda<0$ and $U_{K, \lambda} \in \stackrel{o}{K}$ such that

$$
A^{T} U_{K, \lambda}-\lambda U_{K, \lambda} \in N_{K}(0)
$$

then $A \in \mathcal{L}_{K}^{-}$.

Proof: We set $G:=U_{K, \lambda} \otimes U_{K, \lambda}^{T}$. Note that (32) together with (34) ensure that $U_{K, \lambda} \neq 0$. We have

$$
\langle G x, x\rangle=\left\langle U_{K, \lambda}, x\right\rangle^{2} \geq 0 \quad \forall x \in \mathbb{R}^{n} .
$$

We remark now that

$$
\langle G x, x\rangle>0 \quad \forall x \in K \backslash\{0\} .
$$

Indeed, suppose by contradiction that $\langle G x, x\rangle=0$ for some $x \in$ $K \backslash\{0\}$. Then, $\left\langle U_{K, \lambda}, x\right\rangle=0$ and since $U_{K, \lambda} \in \stackrel{o}{K}$ we obtain from (34) that $x=0$ which is a contradiction. Proposition 1 ensures that $G \in \mathcal{P}_{K}^{++}$since $K$ is a cone. We see also that if $x \in \partial K \subset K$ then $\left(G+G^{T}\right) x=2\left\langle U_{K, \lambda}, x\right\rangle U_{K, \lambda} \in K=$ $K_{\infty}$. Indeed $U_{K, \lambda} \in K,\left\langle U_{K, \lambda}, x\right\rangle \geq 0$ because of (33) and $K$ is a cone. Finally, $\left\langle A x,\left[G+G^{T}\right] x\right\rangle=2\left\langle x, A^{T} U_{K, \lambda}\right\rangle\left\langle U_{K, \lambda}, x\right\rangle$, if $x \in K$ then $\left\langle U_{K, \lambda}, x\right\rangle \geq 0$ and from (35), $\left\langle x, A^{T} U_{K, \lambda}\right\rangle \leq$ $\lambda\left\langle x, U_{K, \lambda}\right\rangle$. It results that $\left\langle A x,\left[G+G^{T}\right] x\right\rangle \leq 2 \lambda\left\langle U_{K, \lambda}, x\right\rangle^{2}=$ $-2|\lambda|\langle G x, x\rangle$.

\section{Examples}

Let us here illustrate the previous results with some simple examples.

Examples 2:

i) Let $K=\mathbb{R}_{+} \times \mathbb{R}_{+} \times \mathbb{R}_{+}$and

$$
A=\left(\begin{array}{ccc}
-1 & -3 & 0 \\
0 & -1 & 0 \\
0 & 0 & -1
\end{array}\right) \text {. }
$$

We see easily that $-A \in \mathcal{P}_{K}^{+}$and thus from Proposition 13, we deduce that $A \in \mathcal{L}_{K}^{-}$.

ii) Let $K=\left\{x \in \mathbb{R}^{2}:-(1 / 2) x_{1} \leq x_{2} \leq-x_{1}\right\}$ and

$$
A=\left(\begin{array}{cc}
0 & 2 \\
-1 & 0
\end{array}\right) \text {. }
$$

Here, $\langle A x, x\rangle=x_{1} x_{2}$ and if $\left(x_{1}, x_{2}\right) \in K \backslash\{0\}$ then $x_{1}<0$ and $x_{2}>0$. It results that $\langle A x, x\rangle<0, \forall x \in$ $K \backslash\{0\}$. Proposition 13 ensures that $A \in \mathcal{L}_{K}^{-}$.

iii) Let $K=\mathbb{R}_{+} \times \mathbb{R}_{+} \times \mathbb{R}_{+}$and

$$
A=\left(\begin{array}{ccc}
-1 & 4 & 0 \\
-1 & -1 & 0 \\
0 & 0 & -1
\end{array}\right) \text {. }
$$

Setting $D=\operatorname{diag}\{1,5,5\}$, we see that $-D A \in \mathcal{P}_{K}^{+}$[see Example 1 vii)] and Proposition 14 ensures that $A \in \mathcal{L}_{K}^{-}$.

iv) Let $K=\mathbb{R}_{+} \times \mathbb{R}_{+}$and

$$
A=\left(\begin{array}{cc}
-1 & -3 \\
0 & 2
\end{array}\right) \text {. }
$$

Then, $\sigma(A)=\{-1,2\}$ and let $\lambda=-1$, we check that

$$
\begin{aligned}
U_{\lambda}=\left(\begin{array}{l}
1 \\
1
\end{array}\right) \in E_{A^{T}}(-1) . \text { Here } \\
U_{\lambda} \otimes U_{\lambda}^{T}=\left(\begin{array}{ll}
1 & 1 \\
1 & 1
\end{array}\right)
\end{aligned}
$$


and thus $x \in \partial K \subset K \Rightarrow\left[U_{\lambda} \otimes U_{\lambda}^{T}\right] x \in K_{\infty}=K$. Moreover $\operatorname{ker}\left\{U_{\lambda} \otimes U_{\lambda}^{T}\right\}=\left\{x \in \mathbb{R}^{2}: x_{2}=-x_{1}\right\}$ so that $K \cap \operatorname{ker}\left\{U_{\lambda} \otimes U_{\lambda}^{T}\right\}=\{0\}$. Using Proposition 15, we obtain that $A \in \mathcal{L}_{K}^{-}$.

Note that the result can also be obtained from Proposition 17 since $U_{\lambda} \in \stackrel{o}{K}, A^{T} U_{\lambda}-\lambda U_{\lambda}=0 \in N_{K}(0)$ and $K$ satisfies the conditions (32)-(34) required in Proposition 17.

v) Let $K=\mathbb{R}_{+} \times \mathbb{R}_{+}$and

$$
A=\left(\begin{array}{cc}
-6 & -6 \\
2 & 2
\end{array}\right)
$$

Then, $\sigma(A)=\{-4,0\}$. Set $\lambda=-4$ and $\mu=0$. We see that $U_{\lambda}=\left(\begin{array}{l}1 \\ 1\end{array}\right) \in E_{A^{T}}(-4)$ and $U_{\mu}=\left(\begin{array}{l}1 \\ 3\end{array}\right) \epsilon$ $E_{A^{T}}(0)$. Here

$$
U_{\lambda} \otimes U_{\mu}^{T}=\left(\begin{array}{ll}
1 & 3 \\
1 & 3
\end{array}\right)
$$

and, thus, $\left\langle\left[U_{\lambda} \otimes U_{\mu}^{T}\right] x, x\right\rangle=x_{1}^{2}+3 x_{2}^{2}+4 x_{1} x_{2}>0$, $\forall x \in K \backslash\{0\}$. Moreover, if $x \in \partial K \subset K$, then

$\left(U_{\lambda} \otimes U_{\mu}^{T}+U_{\mu} \otimes U_{\lambda}^{T}\right) x=\left(\begin{array}{ll}2 & 4 \\ 4 & 6\end{array}\right)\left(\begin{array}{l}x_{1} \\ x_{2}\end{array}\right) \in K_{\infty}=K$.

The result follows from Proposition 15.

\section{Examples 3:}

i) Let $K=\left\{x \in \mathbb{R}^{2}:\|x\| \leq 1\right\}$ and

$$
A=\left(\begin{array}{cc}
-1 & 0 \\
0 & 0
\end{array}\right) \text {. }
$$

The matrix $A$ is neither Lyapunov positive stable on $K$ nor Lyapunov unstable on $K$ in the sense of Definitions 4 and 6 , respectively. Indeed, suppose on the contrary that the matrix $A$ is Lyapunov positive stable on $K$. The structure of $K$ entails that conditions (1) and (2) of Definition 4 are satisfied on $\mathbb{R}^{2}$ and, thus, we obtain the existence of a positive-definite matrix $G$ such that

$$
G A+A^{T} G=\left(\begin{array}{cc}
-2 g_{11} & -g_{12} \\
-g_{21} & 0
\end{array}\right)
$$

is semipositive-definite. A necessary condition for $G$ to be positive definite is $g_{11}>0$. On the other hand, a necessary condition for $G A+A^{T} G$ to be semipositive definite is $g_{11} \leq 0$. The contradiction is obtained. Suppose now that $A$ is unstable on $K$. Here $K_{\infty}=\{0\}$ and condition (3) of Definition 6 implies that $\left\{x \in \mathbb{R}^{2}:\|x\|=1\right\} \subset$ $\operatorname{ker}\left\{G+G^{T}\right\}$. This is a contradiction since condition (1) ensures here that $G+G^{T}$ is nonsingular.

ii) Let $K=\left\{x \in \mathbb{R}^{2}: x_{1} \geq 0, x_{2} \geq 0\right\}$ and

$$
A=\left(\begin{array}{cc}
-1 & 0 \\
0 & 1
\end{array}\right) \text {. }
$$

The matrix $A$ is neither Lyapunov positive stable on $K$ nor Lyapunov unstable on $K$. Indeed, let $G \in \mathbb{R}^{2 \times 2}$ be a strictly copositive matrice on the cone $K$ as required in condition (1) of Definition 4 and Definition 6. Then necessarily $g_{11}>0$ and $g_{22}>0$. On the other hand, we have $\left\langle\left[G A+A^{T} G\right] x, x\right\rangle=-2 g_{11} x_{1}^{2}+2 g_{22} x_{2}^{2}$. Thus, it is clear that neither condition (2) of Definition 4 nor condition (2) of Definition 6 can be satisfied.

Examples 4: Let us here use Theorem 5 to discuss the stability of the trivial solution of Problem (12) and (13) for different matrices $A \in \mathbb{R}^{n \times n}$ and sets $K \subset \mathbb{R}^{n}$ given in Examples 1 and Examples 2. We also discuss the stability of the trivial solution of Problem (12) and (13) without constraint, i.e., with $K=\mathbb{R}^{n}$, and see by the way that the stability of an equilibrium may substantially change as soon as inequality constraints are involved. These results are reported in Table I.

Remark 9: Note that if $K \subset \mathbb{R}^{n}$ is a subspace then the solution $x$ of (12)-(14) satisfies the system

$$
x(t)=P_{K}\left(x(t)-x^{\prime}(t)-A x(t)\right) .
$$

Here, $P_{K}$ is linear, $x(t), x^{\prime}(t) \in K$ and, thus

$$
\frac{d x}{d t}(t)+P_{K} A x(t)=0, \quad \text { a.e. } t \geq t_{0} .
$$

It results that

$$
x(t)=e^{-P_{K} A t} x_{0}
$$

with

$$
e^{-P_{K} A t}=\frac{1}{2 \pi i} \oint_{\Gamma} e^{-t z}\left(z I-P_{K} A\right)^{-1} d z
$$

where $\Gamma$ denotes a Jordan curve enclosing an open disk containing $\sigma\left(P_{K} A\right)$. It is known that variational inequalities, complementarity and projected dynamical systems, are closely related one to each other [37].

\section{APPLICATIONS}

Let us here discuss the stability of a system described by a transfer function $G(s)=C(s I-A)^{-1} B$ and a feedback branch containing a sector static nonlinearity, known as the absolute stability problem [15], [30] (see Fig. 1). Here $A \in \mathbb{R}^{n \times n}, B \in$ $\mathbb{R}^{n \times m}$ and $C \in \mathbb{R}^{m \times n}$. The static nonlinearity $y_{L}=\phi(y)$ is usually assumed to be a locally Lipschitz single-valued function [30, Sec. 10.1], possibly time-varying and piecewise continuous in $t$.

The feedback nonlinearity is here assumed to be a multivalued monotone mapping of the form $\partial \psi_{K}$ where $K \subset \mathbb{R}^{n}$ is a set satisfying conditions $\left(H_{1}\right)-\left(H_{3}\right)$ of Section III, $\psi_{K}$ is the indicator function of $K$ and $\partial$ denotes the convex subdifferential operator. The study of such systems has been initiated in [3]. The state-space equations of such a system are given by: Find $x \in C^{0}\left([0, \infty) ; \mathbb{R}^{n}\right)$ such that $(d x / d t) \in L_{\mathrm{loc}}^{\infty}\left(0,+\infty ; \mathbb{R}^{n}\right)$ and

$$
\begin{aligned}
\frac{d x}{d t}(t) & =A x(t)-B y_{L}(t), \text { a.e. } t \geq 0 \\
y(t) & =C x(t), \quad t \geq 0 \\
y(t) & \in K, \quad t \geq 0 \\
y_{L}(t) & \in \partial \psi_{K}(y(t)), \quad t \geq 0 \\
x(0) & =x_{0} .
\end{aligned}
$$


TABLE I

STABILITY AND INSTABILITY DEPENDING ON $K$

\begin{tabular}{|c|c|c|c|}
\hline $\begin{array}{l}\text { Matrix } A \text { and set } \\
K \text { given in }\end{array}$ & $\sigma(A)$ & $\begin{array}{l}\text { Trivial solution of } \\
\frac{d u}{d t}+A u=0\end{array}$ & $\begin{array}{l}\text { Trivial solution of } \\
\left(\frac{d u}{d t}+A u, v-u\right) \geq 0 \\
\forall v \in K\end{array}$ \\
\hline Example 1 i) & $\{1-\sqrt{2}, 1+\sqrt{2}\}$ & unstable & $\begin{array}{l}\text { Asymptotically } \\
\text { stable }\end{array}$ \\
\hline Example $1 \mathrm{iii)}$ & $\{-4,3\}$ & unstable & $\begin{array}{l}\text { Asymptotically } \\
\text { stable }\end{array}$ \\
\hline Example 1 iv) & $\{1,2\}$ & $\begin{array}{l}\text { Asymptotically } \\
\text { stable }\end{array}$ & $\begin{array}{l}\text { Asymptotically } \\
\text { stable }\end{array}$ \\
\hline Example 1 v) & $\begin{array}{l}\{0.8769-2.0113 i \\
0.8769+2.0113 i \\
1.2463\}\end{array}$ & $\begin{array}{l}\text { Asymptotically } \\
\text { stable }\end{array}$ & $\begin{array}{l}\text { Asymptotically } \\
\text { stable }\end{array}$ \\
\hline Example $1 \mathrm{vi)}$ & $\{0,1-2 i, 1+2 i\}$ & Stable & Stable \\
\hline Example 1 vii) & $\{1,1-2 i, 1+2 i\}$ & $\begin{array}{l}\text { Asymptotically } \\
\text { stable }\end{array}$ & $\begin{array}{l}\text { Asymptotically } \\
\text { stable }\end{array}$ \\
\hline Example 1 viii) & $\left\{0, \frac{3-\sqrt{7} i}{2}, \frac{3+\sqrt{7} i}{2}\right\}$ & Stable & Stable \\
\hline Example $1 \mathrm{ix}$ ) & $\{1,1-2 i, 1+2 i\}$ & $\begin{array}{l}\text { Asymptotically } \\
\text { stable }\end{array}$ & $\begin{array}{l}\text { Asymptotically } \\
\text { stable }\end{array}$ \\
\hline Example $1 \mathrm{x}$ ) & $\{1-\sqrt{3} i, 1+\sqrt{3} i\}$ & $\begin{array}{l}\text { Asymptotically } \\
\text { stable }\end{array}$ & $\begin{array}{l}\text { Asymptotically } \\
\text { stable }\end{array}$ \\
\hline Example 2 i) & $\{-1\}$ & unstable & unstable \\
\hline Example 2 ii) & $\{-\sqrt{2} i, \sqrt{2} i\}$ & Stable & unstable \\
\hline Example 2 iii) & $\{-1,-1-2 i,-1+2 i\}$ & unstable & unstable \\
\hline Example $2 \mathrm{iv}$ ) & $\{-1,2\}$ & unstable & unstable \\
\hline Example $2 \mathrm{v}$ ) & $\{-4,0\}$ & unstable & unstable \\
\hline
\end{tabular}

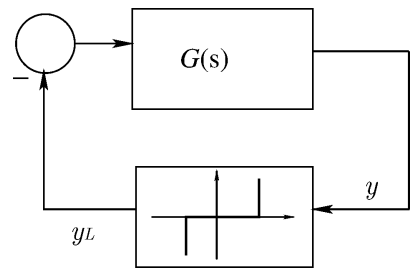

Fig. 1. Absolute stability problem.

Assume there exists a symmetric and invertible matrix $R \in$ $\mathbb{R}^{n \times n}$ such that $R^{-2} C^{T}=B$. Suppose also that there exists

$$
y_{0}:=C R^{-1} x_{0} \in \operatorname{int}\{K\} .
$$

Then, using the change of state vector $z=R x$ and setting

$$
\bar{K}=\left\{h \in \mathbb{R}^{n}: C R^{-1} h \in K\right\}
$$

we see that problem (36)-(40) is equivalent to the following one: find $z \in C^{0}\left([0, \infty) ; \mathbb{R}^{n}\right)$ such that $(d z / d t) \in L_{\mathrm{loc}}^{\infty}\left([0, \infty) ; \mathbb{R}^{n}\right)$ and

$$
\begin{aligned}
\left\langle\frac{d z}{d t}(t)-R A R^{-1} z(t), v-z(t)\right\rangle & \geq 0 \\
\forall v & \in \bar{K}, \text { a.e. } t \geq 0 \\
z(t) & \in \bar{K}, \quad t \geq 0 \\
z(0) & =R x_{0} .
\end{aligned}
$$

Indeed, it suffices to remark that

$$
\begin{gathered}
C x \in K \Leftrightarrow z \in \bar{K} \\
x(0)=x_{0} \Leftrightarrow z(0)=R x_{0}
\end{gathered}
$$

and

$$
\begin{aligned}
\frac{d x}{d t} & \in A x-B \partial \psi_{K}(C x) \Leftrightarrow \\
R \frac{d x}{d t} & \in R A R^{-1} R x-R B \partial \psi_{K}\left(C R^{-1} R x\right) \Leftrightarrow \\
\frac{d z}{d t} & \in R A R^{-1} z-R^{-1} R^{2} B \partial \psi_{K}\left(C R^{-1} z\right) \Leftrightarrow \\
\frac{d z}{d t} & \in R A R^{-1} z-R^{-1} C^{T} \partial \psi_{K}\left(C R^{-1} z\right) \Leftrightarrow \\
\frac{d z}{d t} & \in R A R^{-1} z-\partial \psi_{\bar{K}}(z) .
\end{aligned}
$$

Indeed, $\psi_{\bar{K}}(z)=\left(\psi_{K} \circ C R^{-1}\right)(z)$ and, thanks to (41), we obtain $\partial \psi_{\bar{K}}(z)=R^{-1} C^{T} \partial \psi_{K}\left(C R^{-1} z\right)$. We remark also that the set $\bar{K}$ satisfies the conditions $\left(H_{1}\right)-\left(H_{3}\right)$.

Applying now the results of the Section VI, we may discuss the stability of the trivial solution of (36)-(39).

Corollary 1: Let $K \subset \mathbb{R}^{n}$ be a set satisfying hypothesis $\left(H_{1}\right)-\left(H_{3}\right)$ together with (41) and define $\bar{K}$ as in (42). Suppose that there exists a symmetric and invertible matrix $R \in \mathbb{R}^{n \times n}$ such that $R^{-2} C^{T}=B$. 
i) If $-R A R^{-1} \in \mathcal{L}_{\bar{K}}$, then the trivial equilibrium point of (36)-(39) is stable.

ii) If $-R A R^{-1} \in \mathcal{L}_{\bar{K}}^{++}$, then the trivial equilibrium point of (36)-(39) is asymptotically stable.

iii) Suppose here in addition that $K \backslash\{0\} \neq \emptyset$. If $-R A R^{-1} \in \mathcal{L}_{\bar{K}}^{-}$, then the trivial equilibrium point of (36)-(39) is unstable.

Example 1: Assume that $G(s)=C(s I-A)^{-1} B$, with $(A, B, C)$ a minimal representation, is strictly positive real (SPR), i.e., $r e\{G(i w)\}>0, \forall w \in \mathbb{R}$. From the Kalman-Yakubovitch-Popov Lemma there exist $G=G^{T}$ positive definite and $Q=Q^{T}$ positive definite, such that $G A+A^{T} G=-Q$ and $B^{T} G=C$. Choosing $R$ as the symmetric square root of $G$, i.e., $R=R^{T}$, $R$ positive definite and $R^{2}=G$, we see that $B^{T} R^{2}=C$ and, thus, $R^{-2} C^{T}=B$. Moreover

$$
\langle G A x, x\rangle+\left\langle A^{T} G x, x\right\rangle=-\langle Q x, x\rangle \quad \forall x \in \mathbb{R}^{n} .
$$

Thus

$$
\langle A x, G x\rangle=-\frac{1}{2}\langle Q x, x\rangle \quad \forall x \in \mathbb{R}^{n} .
$$

It results that

$$
-\langle R A x, R x\rangle>0 \quad \forall x \in \mathbb{R}^{n} \backslash\{0\} .
$$

Setting $z=R x$, we see that

$$
-\left\langle R A R^{-1} z, z\right\rangle>0 \quad \forall z \in \mathbb{R}^{n} \backslash\{0\} .
$$

So $-R A R^{-1} \in \mathcal{P}_{\mathbb{R}^{n}}^{++} \subset \mathcal{P}_{\bar{K}}^{++} \subset \mathcal{L}_{\bar{K}}^{++}$. All the conditions of Corollary 1 (part ii) are satisfied and the trivial solution of (36)-(39) is asymptotically stable. The results of [3] with $\varphi=$ $\Psi_{K}$ and [17, Th. 11.2] are here recovered. In case $G(s)$ is positive real (PR) then corollary 1 (part i) applies. As shown in [3] the equilibrium point is unique in this case.

In relation to Proposition 12, we have the following.

Corollary 2: Consider a dissipative linear complementarity system $\dot{x}=A x+B \lambda, 0 \leq y=C x \perp \lambda \geq 0$, with $(A, B, C)$ a PR (resp. SPR) transfer function. Then, Proposition 2 (respectively, Proposition 3 ) applies whatever the state-space representation $w=L x$ with $L \in \mathbb{R}^{n \times n}, L$ nonsingular and $L L^{T}=I$.

Proof: The proof follows from the calculations in (43)-(46) (or using the results in [3] or [17]). Indeed whatever transformation $w=L x$, the above shows that there is always a transformation $z=R w=R L x$ such that the transformed evolution matrix satisfies Proposition 2.

Remark 10: For a relative degree 0 passive LCS $(y=C x+$ $D \lambda, D+D^{T}$ positive definite) the framework in this paper no longer applies since the system is an ordinary differential equation (with Lipschitz continuous single-valued right-hand-side) and no longer an inclusion as in (2). This is easily seen since $\lambda$ is the unique solution of a linear complementarity problem with matrix $\left(\left(D+D^{T}\right) / 2\right)[21]$.

Electrical circuits with ideal diodes and relative degree one between $y$ and $\lambda$ [see (3)] are an example of dissipative systems that fit within this framework with PR or SPR transfer functions $G(s)$ in feedback connection with the corner law [22], [24]. Let us notice that passive LCS have the operator $\lambda \mapsto y$ which is dissipative, but not necessarily not the operator $u \mapsto y$. Hence, passive LCS may not be asymptotically stabilised by output feedback $u=-k y, k>0$, as passive unconstrained systems are [15].

\section{CONCLUSION}

In this paper, the stability of linear evolution variational inequalities is studied. These dynamical systems have unilateral effects, hence, are nonsmooth and nonlinear. The Lyapunov stability is considered, and Lyapunov's second method is investigated. It is shown that the extension is nontrivial, and that the stability of the unconstrained system may drastically differ from that of the constrained system. Examples are given to illustrate the developments, as well as criteria which allow one to test the stability (or the instability). Links to other classes of hybrid dynamical systems (like differential inclusions, complementarity systems) are provided.

\section{REFERENCES}

[1] A. Berman, M. Neumann, and R. J. Stern, Nonnegative Matrices in Dynamic Systems. New York: Wiley, 1989.

[2] F. Blanchini, "Set invariance in control," Automatica, vol. 35, no. 11, pp. 1747-1767, 1999.

[3] B. Brogliato, "Absolute stability and the Lagrange-Dirichlet theorem with monotone multivalued mappings," Syst. Control Lett., vol. 51, no. 5, pp. 343-353, 2004.

[4] - "On the control of nonsmooth complementarity dynamical systems," Philo. Trans. Royal Soc., ser. A, vol. 359, no. 1789, pp. 2369-2384, 2001.

[5] - "Some perspectives on the analysis and control of complementarity systems," IEEE Trans. Automat. Contr., vol. 48, pp. 918-935, June 2003.

[6] D. Goeleven, M. Motreanu, and V. Motreanu, "On the stability of stationary solutions of evolution variational inequalities," Adv. Nonlinear Variational Inequalities, vol. 6, pp. 1-30, 2003.

[7] D. Hershkowitz and N. Keller, "Positivity of principal minors, sign symmetry and stability," Linear Alg. Applicat., vol. 364, pp. 105-124, 2003.

[8] J. P. Hespanha and A. S. Morse, "Stabilization of nonholonomic integrators via logic-based switching," Automatica, vol. 35, no. 3, pp. 385-394, 1999.

[9] J. A. Ball, M. V. Day, T. Yu, and P. Kachroo, "Robust $L_{2}$-gain control for nonlinear systems with projection dynamics and input constraints: An example from traffic control," Automatica, vol. 35, no. 3, pp. 429-444, 1999.

[10] P. Quittner, "On the principle of linearized stability for variational inequalities," Math. Ann., vol. 283, pp. 257-270, 1989.

[11] _ - "On the stability of stationary solutions of parabolic variational inequalities," Czech. Math. J., vol. 40, pp. 472-474, 1990.

[12] _ "An instability criterion for variational inequalities," Nonlinear Anal. TMA, vol. 15, pp. 1167-1180, 1990.

[13] N. Rouche and J. Mawhin, Equations Différentielles Ordinaires. Paris, France: Masson and Cie, 1973, vol. 2.

[14] N. Rouche, P. Habets, and M. Laloy, Stability Theory by Liapunov's Direct Method. New York: Springer-Verlag, 1977.

[15] R. Lozano, B. Brogliato, O. Egeland, and B. Maschke, Dissipative Systems Analysis and Control. London, U.K.: Springer-Verlag, 2000.

[16] A. van der Schaft and H. Schumacher, An Introduction to Hybrid Dynamical Systems. London, U.K.: Springer-Verlag, 2000, vol. LNCIS 251.

[17] K. Camlibel, W. P. M. H. Heemels, and H. Schumacher, "On linear passive complementarity systems," Eur. J. Control, vol. 8, no. 3, pp. 220-237, 2002.

[18] W. P. M. H. Heemels, H. Schumacher, and S. Weiland, "Linear complementarity systems," SIAM J. Appl. Math., vol. 60, no. 4, pp. 1234-1269, 2000.

[19] J. B. Hiriart-Urruty and C. Lemaréchal, Fundamentals of Convex Analysis, Springer Grundlehren Text Editions ed. Berlin, Germany: Springer-Verlag, 2001. 
[20] D. Goeleven, D. Motreanu, Y. Dumont, and M. Rochdi, Variational and Hemivariational Inequalities-Theory, Methods and Applications. Vol. 1: Unilateral Analysis and Unilateral Mechanics, ser. Nonconvex Optimization and its Applications. Norwell, MA: Kluwer, 2003, vol. 69.

[21] K. G. Murty. (1997) Linear Complementarity, Linear and Nonlinear Programming [Online]. Available: http://www-personal.engin.umich.edu/ murty/book/LCPbook/

[22] B. Brogliato, Nonsmooth Mechanics, 2nd ed. London, U.K.: SpringerVerlag, 1999.

[23] M. Frémond, Non-Smooth Thermomechanics. Berlin, Germany: Springer-Verlag, 2002.

[24] Ch. Glocker, Set Valued Force Laws. London, U.K.: Springer-Verlag, 2001, vol. LNAM 1, Lecture Notes in Applied Mechanics.

[25] R. A. Horn and C. R. Johnson, Topics in Matrix Analysis. Cambridge, U.K.: Cambridge Univ. Press, 1994.

[26] P. Lancaster and M. Tismenetsky, The Theory of Matrices, 2nd ed. New York: Academic, 1985.

[27] M. Kunze and M. M. Marques, "An introduction to Moreau's sweeping process," in Impacts in Mechanical Systems, B. Brogliato, Ed. New York: Springer-Verlag, 2000, vol. LNP 551, Lecture Notes in Physics, pp. 1-60.

[28] A. A. ten Dam, E. Dwarshuis, and J. C. Willems, "The contact problem for linear continuous-time dynamical systems: A geometric approach," IEEE Trans. Automat. Contr., vol. 42, pp. 458-472, Apr. 1997.

[29] M. Vassilaki and G. Bitsoris, "Constrained regulation of linear continuous-time dynamical systems," Syst. Control Lett., vol. 13, pp. 247-252, 1989.

[30] H. K. Khalil, Nonlinear Systems, 2nd ed. Upper Saddle River, NJ: Prentice-Hall, 1996.

[31] M. Vidyasagar, Nonlinear Systems Analysis, 2nd ed. Upper Saddle River, NJ: Prentice-Hall, 1993.

[32] D. Goeleven, G. E. Stravoulakis, G. Salmon, and P. D. Panagiotopoulos, "Solvability theory and projection methods for a class of singular variational inequalities: Elastostatic unilateral contact applications," $J$. Optim. Theory Applicat., vol. 95, no. 2, pp. 263-293, 1997.

[33] J. C. Yao, "Variational inequalities with generalized monotone operators," Math. Oper. Research, vol. 19, no. 3, pp. 691-705, 1994

[34] K. H. Johansson, A. Rantzer, and K. J. Astrom, "Fast switches in relay feedback systems," Automatica, vol. 35, no. 4, pp. 539-552, 1999.

[35] A. N. Michel and B. Hu, "Toward a stability theory of general hybrid systems," Automatica, vol. 35, no. 3, pp. 371-384, 1999.

[36] A. N. Michel, K. Wang, and B. Hu, Qualitative Theory of Dynamical Systems, 2nd ed. New York: Marcel Dekker, 2001

[37] W. P. M. H. Heemels, J. M. Schumacher, and S. Weiland, "Projected dynamical systems in a complementary formalism," Oper. Res. Lett., vol. 27, pp. 83-91, 2000.

[38] P. Dupuis and A. Nagurney, "Dynamical systems and variational inequalities," Ann. Oper. Res., vol. 44, pp. 9-42, 1993.

[39] A. Nagurney and D. Zhang, "Projected dynamical systems and variational inequalities with applications," in Int.Series in Operations Research and Management Sciences. Norwell, MA: Kluwer, 1996, vol. 2.
[40] D. Zhang and A. Nagurney, "On the stability of projected dynamical systems," J. Optim. Theory Applicat., vol. 85, no. 1, pp. 97-124, 1995.

[41] T. L. Friesz, D. Bernstein, and R. Stough, "Dynamic systems, variational inequalities and control theoretic models for predicting time-varying urban network flows," Transport. Sci., vol. 30, no. 1, pp. 14-31, Feb. 1996.

[42] T. L. Friesz, D. Bernstein, T. E. Smith, R. L. Tobin, and B. W. Wie, "A variational inequality formulation of the dynamic network user equilibrium problem," Oper. Res., vol. 41, no. 1, pp. 179-191, Jan./Feb. 1993.

[43] M. J. Smith, "The existence, uniqueness and stability of traffic equilibria," Transport. Res., vol. 13B, pp. 295-304, 1979.

[44] — , "The stability of a dynamic model of traffic assignment: An application of a method of Lyapunov," Transport. Sci., vol. 18, pp. 245-252, 1984

[45] A. Nagurney and D. Zhang, "Projected dynamical systems in the formulation, stability analysis, and computation of fixed-demand traffic network equilibria," Transport. Sci., vol. 31, no. 2, pp. 147-158, May 1997.

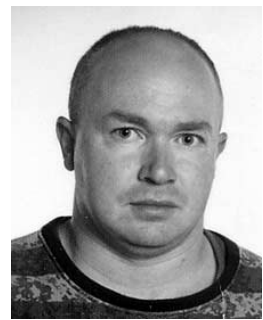

Daniel Goeleven was born in 1965 in Bruxelles, Belgium. He graduated in applied mathematical engineering from the Université Catholique de Louvain, Louvain-La-Neuve, Belgium, in 1989, and received the Ph.D. degree in mathematics from the Facultés Universitaires de Namur, Belgium, in 1993.

He was an FNRS Researcher for two years and he held an Alexander von Humboldt postdoctoral fellowship at the RWTH Aachen, Germany, in 1996. Since that time, he has been a Professor at the Université de La Reunion, France. His major scientific interests concern variational inequalities and nonsmooth mechanics. He wrote Noncoercive Variational Problems and Related Results (Pitman Research Notes in Mathematics Series, No357, Longman, 1996), and coauthored with three colleagues Variational and Hemivariational Inequalities. Theory, Methods and Applications (Norwell, MA: Kluwer, 2003).

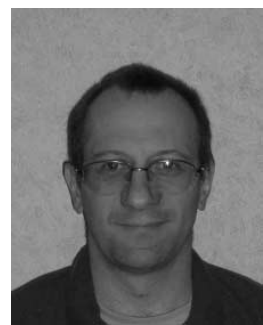

Bernard Brogliato was born in 1963. He graduated from the Ecole Normale Supérieure de Cachan, Paris, France, and received the Ph.D. degree in systems and control from the Institut National Polytechnique de Grenoble, Grenoble, France, in January 1991.

Currently, he is with the French National Institute for Computer Science and Control (INRIA), in the Bipop Project. His main scientific interests are in nonsmooth dynamical systems modeling, analysis, and control, as well as dissipative systems. He wrote the monograph Nonsmooth Mechanics (London, U.K.: Springer, 1999) and coauthored Dissipative Systems Analysis and Control (London, U.K.: Springer, 2000). 